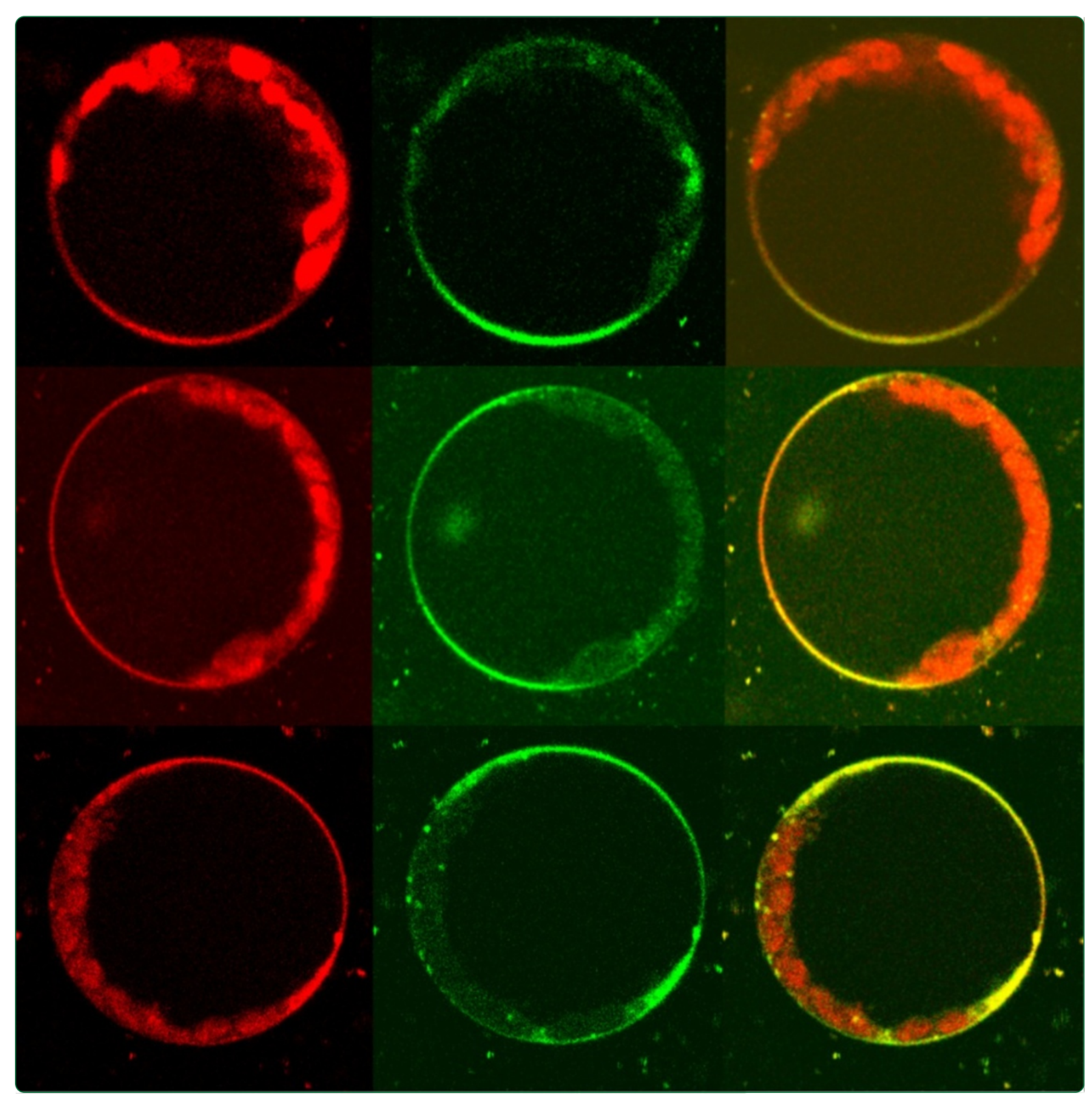

Methods of staining and visualization of sphingolipid enriched and non-enriched plasma membrane regions of Arabidopsis thaliana with fluorescent dyes and lipid analogues

Blachutzik et al. 


\title{
Methods of staining and visualization of sphingolipid enriched and non-enriched plasma membrane regions of Arabidopsis thaliana with fluorescent dyes and lipid analogues
}

\author{
Jörg O Blachutzik ${ }^{1,2}$, Fatih Demir ${ }^{1,3}$, Ines Kreuzer ${ }^{1}$, Rainer Hedrich ${ }^{1}$ and Gregory S Harms ${ }^{2,4^{*}}$
}

\begin{abstract}
Background: Sterols and Sphingolipids form lipid clusters in the plasma membranes of cell types throughout the animal and plant kingdoms. These lipid domains provide a medium for protein signaling complexes at the plasma membrane and are also observed to be principal regions of membrane contact at the inception of infection. We visualized different specific fluorescent lipophilic stains of the both sphingolipid enriched and non-sphingolipid enriched regions in the plasma membranes of live protoplasts of Arabidopsis thaliana.
\end{abstract}

Results: Lipid staining protocols for several fluorescent lipid analogues in plants are presented. The most emphasis was placed on successful protocols for the single and dual staining of sphingolipid enriched regions and exclusion of sphingolipid enriched regions on the plasma membrane of Arabidopsis thaliana protoplasts. A secondary focus was placed to ensure that these staining protocols presented still maintain cell viability. Furthermore, the protocols were successfully tested with the spectrally sensitive dye Laurdan.

Conclusion: Almost all existing staining procedures of the plasma membrane with fluorescent lipid analogues are specified for animal cells and tissues. In order to develop lipid staining protocols for plants, procedures were established with critical steps for the plasma membrane staining of Arabidopsis leaf tissue and protoplasts. The success of the plasma membrane staining protocols was additionally verified by measurements of lipid dynamics by the fluorescence recovery after photobleaching technique and by the observation of new phenomena such as time dependent lipid polarization events in living protoplasts, for which a putative physiological relevance is suggested.

Keywords: Protoplasts, Lipid polarization, Lipophilic fluorescent dyes, Laurdan, Sphingolipid, Liquid (dis-) ordered phase, Plasma membrane, Fluorescence microscopy

\section{Background}

Lipid domains in the plasma membrane (PM) help to cluster proteins in eukaryotic cells. They serve as transient domains for the attachment of proteins mainly involved in signal transduction, especially from cells from the animal kingdom [1]. More recently, much

\footnotetext{
*Correspondence: gregory.harms@imaging-microscopy.com

${ }^{2}$ Microscopy Group, Rudolf Virchow Center, University of Würzburg, Josef

Schneider Str. 2, D15, D-97080 Würzburg, Germany

${ }^{4}$ Departments of Biology and Physics, Wilkes University, 84 W. South St., Wilkes-Barre, PA 18766, USA

Full list of author information is available at the end of the article
}

evidence has accumulated to indicate that membrane domains play important roles in the defense against pathogens and also in the cell signaling in plants [2]. Lipid domains, also called "lipid rafts", in the plasma membrane are spatially organized platforms structurally defined by an enrichment of sterols and sphingolipids $[3,4]$. From a historical perspective, individual lipid components within membrane domains are biochemically associated according to their resistance to treatment with mild non-ionic detergents such as Triton X-100 [5] and are commonly referred to as detergent-resistant membranes (DRMs). Detergent treatment leads to

\section{() Biomed Central}


artifacts, and detergent resistance does not necessarily imply lipid rafts $[6,7]$. However, the enrichment of a specific protein in DRMs suggests an affinity for a distinct lipid environment and indicates localization in membrane subfractions that correlate to lipid nanodomains [8], but DRMs are purely biochemical in nature and do not allow one to specifically observe the domains on the plasma membrane. It is now suggested that only through the direct observation of the domains on the plasma membrane by microscopy one can truly make the claim of "lipid raft" [9]. Lipid rafts have primarily been characterized in the animal kingdom as nanodomains in the plasma membrane where they serve as signalling platforms [1]. Following the concept that these sterol- and sphingolipid-enriched structures are highly dynamic in position and composition it is assumed that individual nanodomains can be stimulated to coalesce into larger, more stable domains by specific lipid-lipid, lipid-protein and protein-protein oligomerizing interactions [10]. In native plasma membranes of plants and fungi similar structures of high lipid order seem to exist, leading towards a lateral segregation of membrane components [8,11]. In plants and fungi plasma membrane domains appear to be quite stable in location, exhibiting less lateral mobility in the bilayer than the lipid raftcounterpart in animals; therefore, these structures should be referred to as membrane (micro-) domains to better distinguish between membrane domains in animals and plants/fungi [9]. Especially in fungi there are striking hints that point towards the coexistence of different membrane compartments of individual protein composition [11,12].

The observation of lipid raft domains in animal cells through fluorescent lipid analogues has produced key results towards the essential knowledge that i) the domains are of the liquid ordered phase whereas the nondomain regions tend to be in the liquid disordered phase, ii) the domains have distinctly smaller sizes and relatively lower mobility than the non-domain regions and iii) the fluorescent lipid analogues specific to the domains tend to specifically appear in the DRM fraction, creating a link from biochemical to microscopic observation.

Among the proteins found in DRM fractions isolated from eukaryotic plasma membranes GPI-anchored proteins as well as subsets of membrane attached proteins involved in signaling were enriched, implicating a physiological importance for the clustering of sphingolipids and sterols $[7,13,14]$. These lipid assemblies seem to be essential for eukaryotic systems. In vital eukaryotic nerve cells sterol depletion experiments resulted in a depletion of ligand-dependent activation of receptor tyrosine kinases at the plasma membrane ([15] and references therein). In yeast it was shown that several membrane subcompartments exist in which proton-coupled amino acid/nucleotide/sugar transporters, sphingolipids and sterols accumulated [12]. In plants membrane patches could be visualized by expressing a fluorescently tagged Remorin protein from potato that showed a strong sterol-dependency in vitro $[2,16]$. The biochemical identification of lipid and protein components in plasma membrane domains has been investigated particularly in mammalian cell types and progressively in yeast but yet inert in plants $[9,17]$. Despite the experimental evidence in the plant kingdom that sterol-dependent proteins exist, there have been, to our knowledge, no efforts been made to selectively label liquid (dis-) ordered membrane compartments in viable plant cells using combinations of fluorescent dyes and lipid analogues. This might have to do with the fact that most of the commercially available dyes suited for confocal microscopy are specifically designed to stain the plasma membranes of animals and not have been reliably shown for plants.

Like that of animals, the plant plasma membrane is laterally segregated. Membrane domains show a lower lateral mobility than their lipid raft-counterparts in animals but also differ regarding the lipid composition, most notably with respect to the complex sterol profile. In Arabidopsis the most abundant sterols are cholesterol, sitosterol, stigmasterol and campesterol, while cholesterol is the predominating sterol in vertebrate plasma membranes [18]. The overall lipid composition in Arabidopsis consists of $37.7 \mathrm{~mol} \%$ sterols, $15.6 \mathrm{~mol} \%$ glycolipids (among them 6.8 mol\% glycosphingolipids) and $46.7 \mathrm{~mol} \%$ phospholipids [19]. More recently it was estimated that the content of sphingolipids within the plasma membrane of plants could make up to $>40 \%$ [20]. Due to their complex sterol profile plant plasma membranes might be less sensitive towards thermal shocks compared to animal systems [18].

Hence, if physiological plant plasma membranes are subdivided into sphingolipid enriched and nonsphingolipid enriched regions, a clearly different picture from the animal cell view might emerge. Individual lipid domains might show a wider variety of sterol-/sphingolipid assemblies - due to the complex sterol profile of plants. However, it could also be conceivable that the whole plant plasma membrane might function as a liquid ordered compartment at macro scale.

From experiments using model membranes there is proof that different lipid phases can coexist, depending on the lipid composition of the system and on temperature [21,22]. At low temperatures lipid mixtures consisting of solely glycerophospholipids and sphingolipids appear to exist as gel-phase $\left(\mathrm{L}_{\beta}\right)$; in the $\mathrm{L}_{\beta}$-phase the hydrophobic lipid tails are in the all-trans position fully expanded and are accordingly strong and lipids are densely packed. As a consequence lipids resident in $\mathrm{L}_{\beta}$ phases hardly can move. With increasing temperature 
glycerophospholipids and sphingolipids show a phase transition and appear to exist as fluid-crystalline phase $\left(\mathrm{L}_{\alpha}\right)$. In the $\mathrm{L}_{\alpha}$-phase attractive Van-der-Waals forces are decreasing and lipids exhibit a higher degree of lateral movement $[23,24]$. Phase transitions induce changes in the order of the system. For each lipid species the phase transition temperature is defined [25]. If cholesterol is added to lipid mixtures consisting of glycerophospholipids and sphingolipids, another phase is forming, that reflects an intermediate between $L_{\alpha^{-}}$and $L_{\beta}$-phases. This phase is called liquid ordered $\left(\mathrm{L}_{\mathrm{o}}\right)$-phase [24]. At the same time the loosely packed lipids of the $\mathrm{L}_{\alpha}$-Phase form the liquid disordered $\left(\mathrm{L}_{\mathrm{d}}\right)$-phase [26]. In ternary lipid mixtures consisting of phosphatidylcholin, sphingomyelin and cholesterol $\mathrm{L}_{\mathrm{o}}$-phases were enriched in sphingomyelin and cholesterol, while $\mathrm{L}_{\mathrm{d}}$-phases mainly consisted of phosphatidylcholin $[27,28]$. Sterols enhanced the existence of $\mathrm{L}_{\mathrm{o}}$-phases since they lower the enthalpy of the $\mathrm{L}_{\beta} / \mathrm{L}_{\alpha}$-phase transition. These differences in enthalpy disappeared at a cholesterol content of $50 \mathrm{~mol} \%$ [26]. Nevertheless, the basic mechanisms enabling lipid phase transitions are not yet fully understood $[29,30]$.

Here, different lipid compartments in the plasma membranes of living plant tissues and cells have been visualized using one- and two-photon microscopy. Beyond establishing simple and successful staining protocols for FM4-64, LRB-PE, DilC 12 , DiIC 18 , DiD, Bodipy-labeled $C_{12}$ Sphingomyelin (BD-SM) and Laurdan for plant cells, we observed evidence for the existence of different lipid phases in protoplasts that are reminiscent of lipid ordered/lipid disordered phases in model membranes. These findings were strengthened by accompanying lipid dynamics of the individual lipid phases and by means of the relative degree of lipid order, as documented by Laurdan. Laurdan imaging demanded an employment of two-photon microscopy to excite fluorescent naphthalene moieties.

\section{Results}

Based on experimental findings from experiments on model membranes and mammalian tissues we modified common staining protocols of the florescent probes FM4-64 (Figure 1A and B; Figure 2A-D), LRB-PE (Figure $2 \mathrm{E}$ and $\mathrm{F}$ ), $\mathrm{DiIC}_{12}$ (Figure $2 \mathrm{G}$ and $\mathrm{H}$ ), $\mathrm{DiIC}_{18}$ (Figure 2I and J), DiD (Figure $2 \mathrm{~K}$ and $\mathrm{L}$ ), BD-SM (Figure $1 \mathrm{C}$ and $\mathrm{D}$; Figure $\mathrm{BA}$ and $\mathrm{B}$ ) and Laurdan (Figure 4) for their use in plants and documented their plasma membrane participation in vivo. Beyond the performance of single staining experiments of epidermal tissues (Figure 1), we optimized protocols for dual staining experiments on protoplasts (Figure 5). Dual staining was performed to create an assay by fluorescence microscopy to observe if other membrane constituents colocalize or segregate from sphingolipid-rich environments upon fluorescence labeling. The results presented here

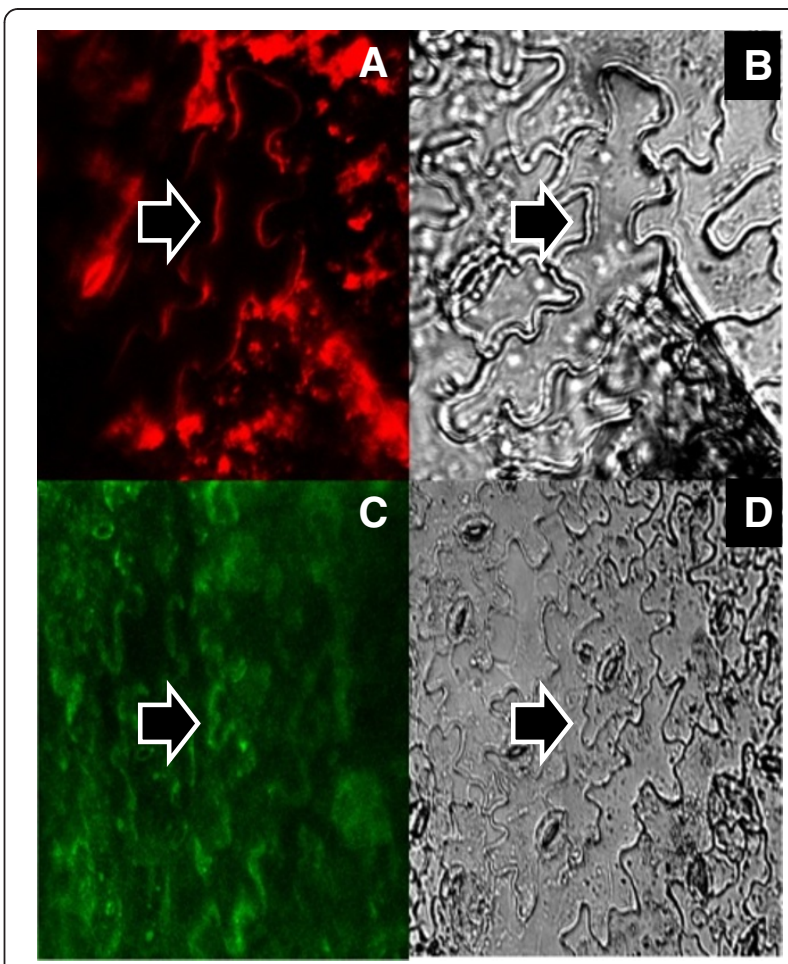

Figure 1 Whole tissue staining of $A$. thaliana epidermal strips. Whole tissue staining of $A$. thaliana epidermal strips, using FM4-64 and BD-SM; (A) FM4-64 stain; according to the white light image (B) some of the fluorescent structures imaged were of the plasma membrane (arrows); (C) BD-SM stain; compared to the white light image (D) a successful plasma membrane staining was achieved (arrows). Nevertheless, strong background fluorescence signals did not allow for proper imaging using epidermal strips.

indicate that the dual staining separately labeled different lipid phases on individual cells. Hence we establish staining protocols suited for plant tissues and protoplasts, using commercially available fluorescent dyes, to not only describe the lipid segregation phenomenon in plants but also to establish a non-biochemical assay of protein and lipid colocalization. All staining protocols, dyespecific excitation/emission wavelengths and proper adjustments of the laser scanning microscopes are described in the methods section.

\section{Whole tissue staining}

Using the standard manufacturer's protocols, plant cells and tissues did not survive the staining procedures as observed by cell morphology and by trypan blue staining presumably due to the high content of chloroform, alcohols and dimethylsulfoxide (DMSO). Thus, new strategies employing large dilutions of DMSO-stocks of florescent probes in aqueous solutions as well as the use of longer incubation times at room temperature (up to $4 \mathrm{~h}$ ) and at $4^{\circ} \mathrm{C}$ (more than $4 \mathrm{~h}$ ) displayed good staining results 


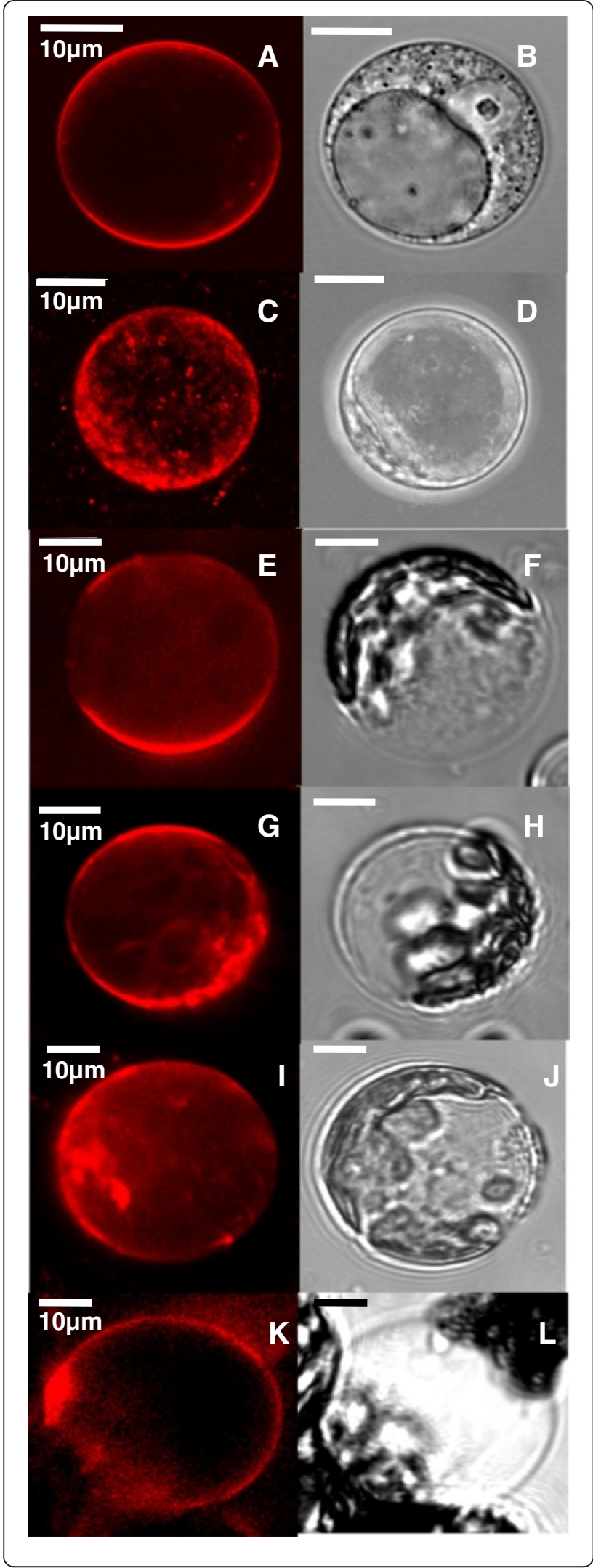

Figure 2 Single dye staining of protoplasts. Single dye staining of protoplasts, using FM4-64 (A; C), LRB-PE (E), DilC $12(\mathbf{G})$, DilC 18 (I), and $\operatorname{DiD}(\mathbf{K})$; all dyes appeared at the plasma membrane. Upon FM4-64 treatment first fluorescent vesicles appeared in the cytoplasm within 20 minutes; endocytosis events were strongly enhanced with ongoing incubation time (C; 30 minutes post FM4-64 incubation). All staining protocols were designed with respect to cell viability; macroscopically the protoplasts were not affected and maintained their round shape $(\mathbf{B} ; \mathbf{D} ; \mathbf{F} ; \mathbf{H} ; \mathbf{J} ; \mathbf{L})$. Viability was successfully tested using trypan blue.

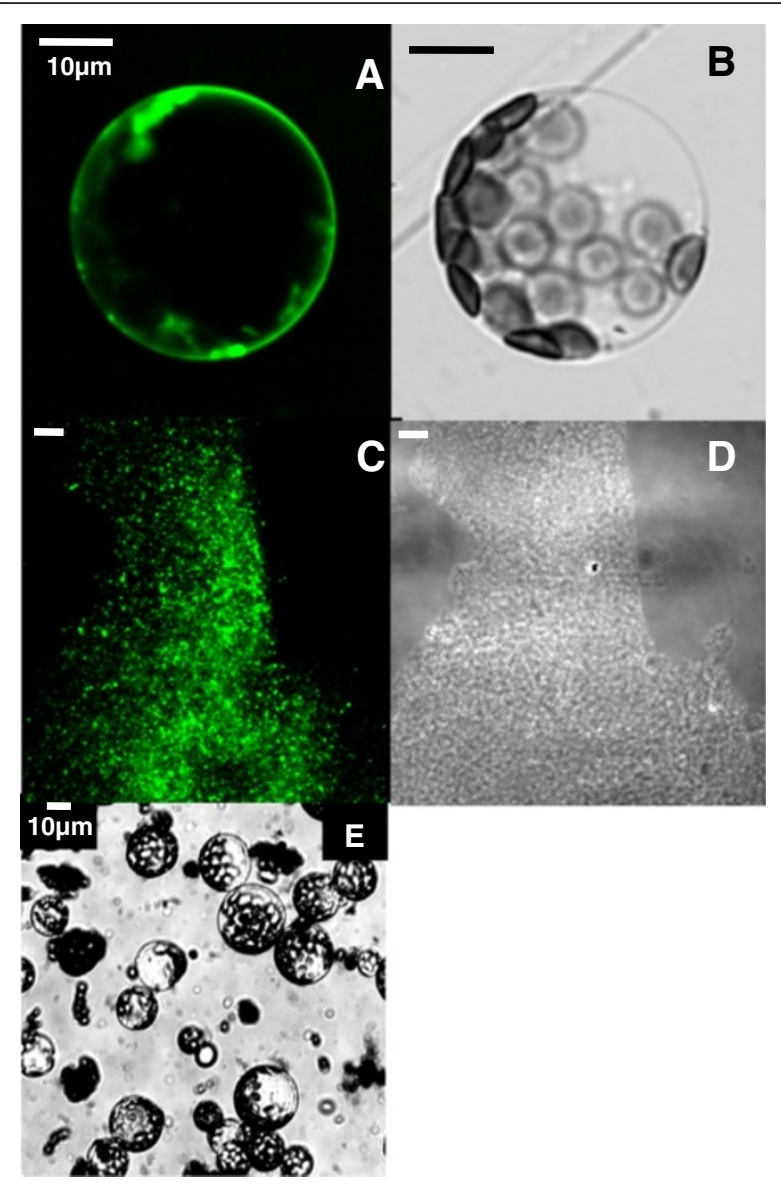

Figure $3 \mathrm{BD}-\mathrm{SM}$ at the plasma membrane and in the DRMfraction. BD-SM appearance at the plasma membrane and in the DRM-fraction. (A \& B) BD-SM reliably stained plasma membranes in protoplasts without losing cell viability. Fluorescence $(\mathbf{A})$ and transmission image of (B) a BD-SM stained protoplast. BD-SM fluorescence was specific to the plane of the membrane $(\mathbf{C} \& \mathbf{D})$. When applied to purified plasma membranes BD-SM appeared in the Arabidopsis DRM-fraction. Fluorescence $(\mathbf{C})$ and transmission image (D) of purified membranes from the DRM-fraction. (E) is showing a bunch of protoplasts after trypan blue treatment; trypan blue is excluded from the cytosol of intact cells (here shown after FM4-64 treatment; in protoplasts treated with $L R B-P E$, DilC 12 , DilC ${ }_{18}$, DiD, BD-SM and Laurdan similar results were obtained using trypan blue). 


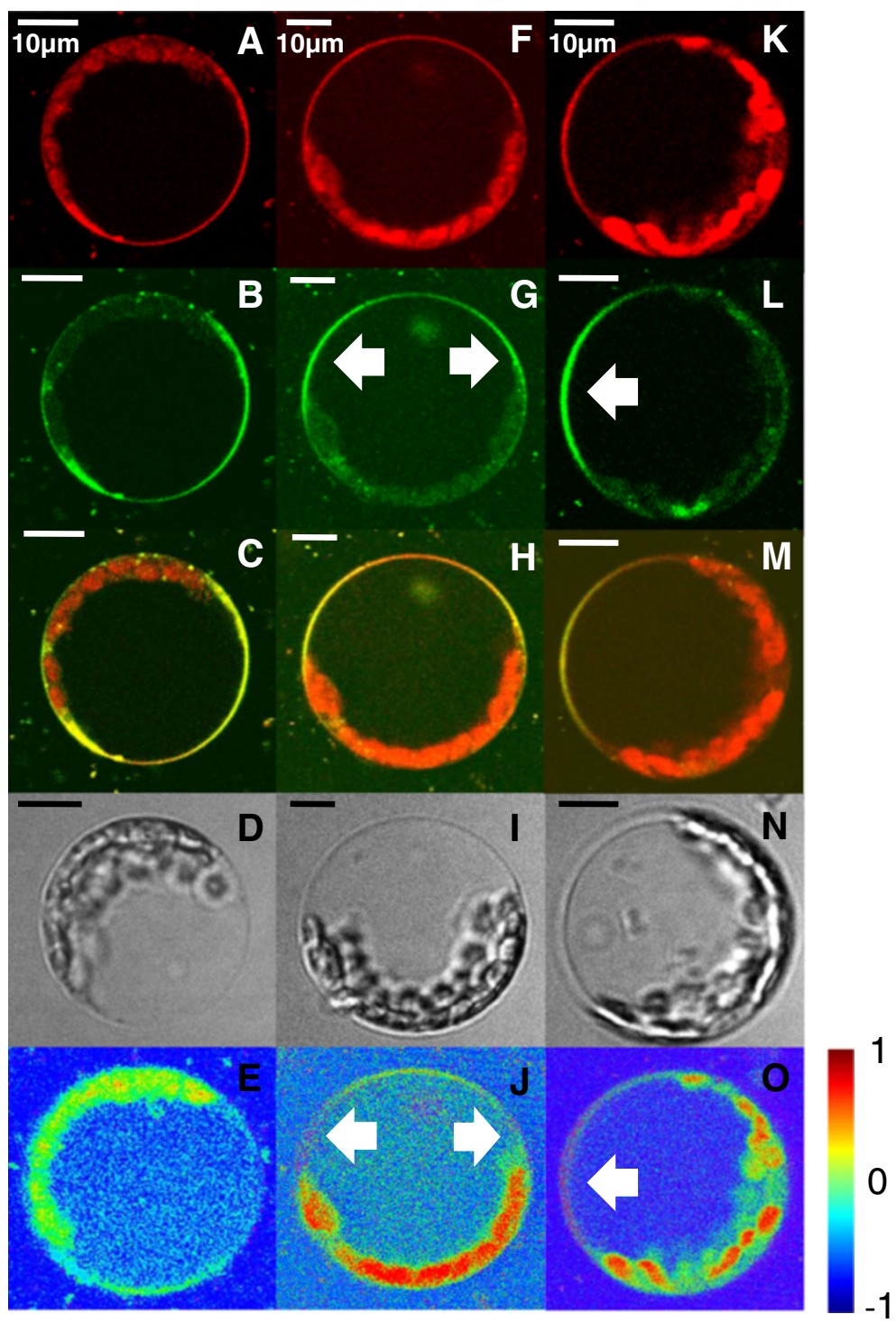

Figure 4 Time-dependent polarization of protoplasts with Laurdan. Time-dependent polarization of protoplasts as indicated by Laurdan; the more red shifted emission spectrum of Laurdan $(\mathbf{A} ; \mathbf{F} ; \mathbf{K})$ indicated polar phases of higher water content, whereas a more blue shifted emission spectrum (here: green, false color; $\mathbf{B} ; \mathbf{G} ; \mathbf{L})$ indicated apolar phases harboring less water; $(\mathbf{C} ; \mathbf{H} ; \mathbf{M})$ : merged images; (E; J; $\mathbf{O})$ : $G P$-values, corresponding to the GP-scale (-1: pure water phase; +1 : fully ordered phase). (A-D) in freshly isolated protoplasts there was no polarization detectable, GP-values ranged from - 0.3 to $0.2(\mathbf{E}) ;(\mathbf{F}-\mathbf{J}) 15 \mathrm{~h}$ post cell wall removal a lipid polarization was detected; at the lateral sides of the membrane areas of high lipid order emerged (J, arrows); $(\mathbf{K}-\mathbf{N}) 20 \mathrm{~h}$ post cell wall removal a wide part of the plasma membrane appeared as polarized ( $\mathbf{O}$, arrow), accompanied by GP-values of up to 0.8 .

without causing any significant levels of cell death. Strong background fluorescence signals, especially for FM4-64 (Figure 1A) and BD-SM (Figure 1C), did not allow for proper imaging of individual cells. Instead fuzzy structures were seen which resulted from accumulations of the dyes within the microfibril textures of cell walls and from unspecific leaf autofluorescence signals (Figure 1). Some of the structures were of the plasma membrane, which we could verify by imaging with fluorescence protein-tagged membrane proteins in transgenic plant lines (data not shown), thus verifying previously recorded plant plasma membrane staining with FM4-64 [31]. To obtain good staining results cell wall components were enzymatically removed (see methods section).

\section{Single dye staining of protoplasts}

The generation of protoplasts not only allowed better accessibility of the PM for the application of individual lipid analogues from the extracellular side but also helped to reduce unspecific fluorescence signals, which 

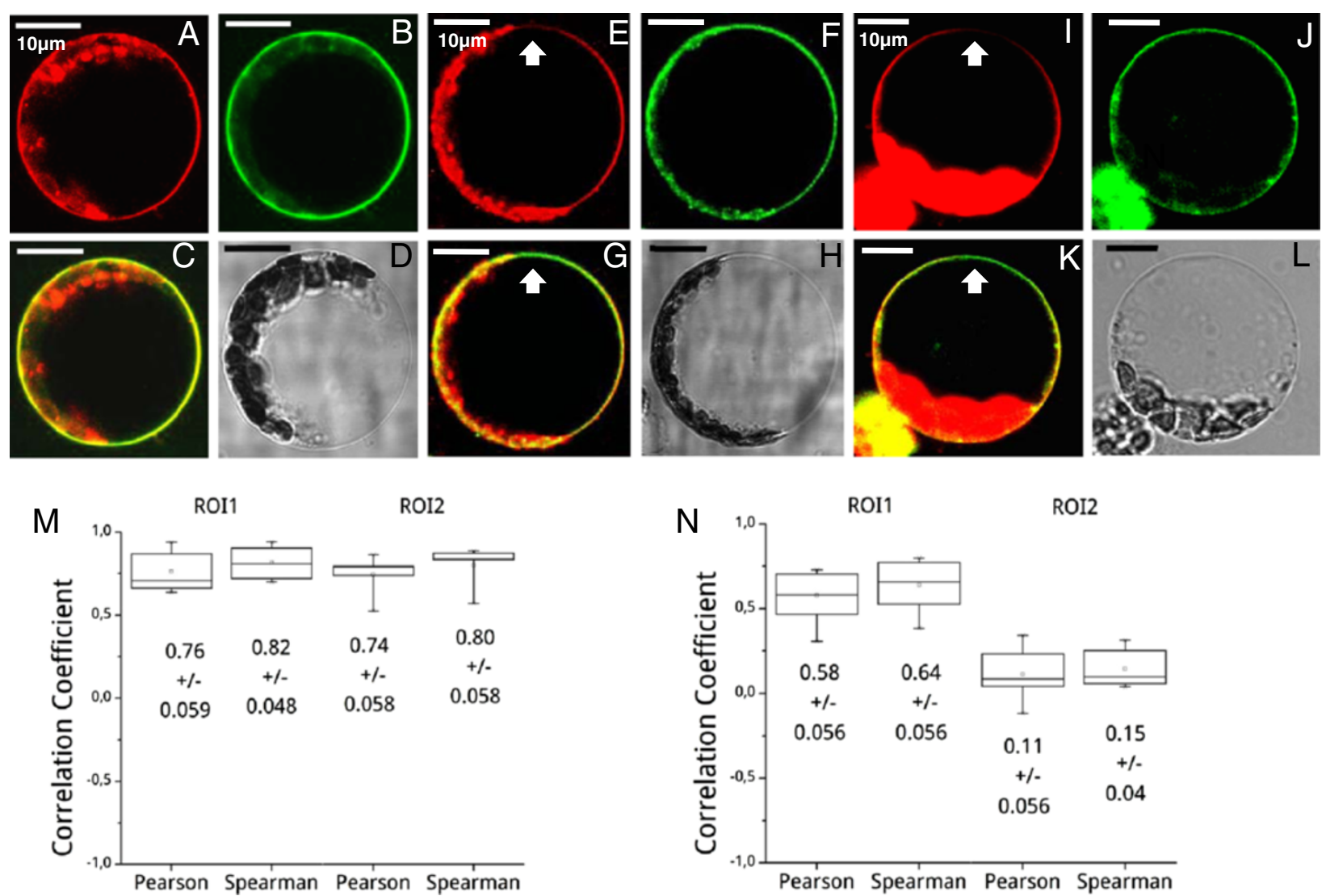

Figure $\mathbf{5}$ Combined dye staining of protoplasts and correlation analyses. There was no polarization detectable in freshly isolated, intact protoplasts. FM4-64 (A) \& BD-SM (B) appeared to be homogeneous in the plasma membrane as viewed in the merged image (C) of the protoplast (transmission image (D)). (E-F)The occurrence of lipid polarization was observed 15 h post cell wall removal; (E) FM4-64 fluorescence, (F) BD-SM fluorescence; at the dorsal side there was a depletion of the FM4-64 fluorescence signal detected (E, $\mathbf{G}$ (merged image of $\mathbf{E}$ and $\mathbf{F})$; arrows) in a viable protoplast ( $\mathbf{H}$ (transmission image of the protoplast)). In $15 \mathrm{~h}$ old protoplasts these findings were confirmed by a combined use of LRB-PE (I) and BD-SM (J). A depletion of the LRB-PE fluorescence signal was also detected (I, K (merged image of $\mathbf{I}$ and $\mathbf{J}$ ); arrows) in intact protoplasts (L (transmission image)). (M): Pearson and Spearman correlation coefficients resulting from two independent ROls in freshly isolated, FM4-64/BD-SM treated protoplasts. Both coefficients indicated a colocalization of fluorescence signals. ( $n=10$ protoplasts). (N): In 15 h old protoplasts the determined correlation coefficients showed a decrease between unpolarized regions (ROI1) and polarized regions of the plasma membrane (ROI2). For ROI1 unpolarized regions were determined. ROI2 reflects the correlation of green BD-SM and red FM4-64 fluorescence signals next to ROl1 in unpolarized regions in (M) and in polarized regions (FM4-64 depleted) in (N) of the plasma membrane ( $n=10$ protoplasts). (See methods and Figure S2 for details of the analysis in $\mathbf{M} \& \mathbf{N}$ ).

we mostly attribute to the unspecific staining of the cell wall and its components. The successful staining protocols were tailor-made for each lipid analogue dye. In each case, we verified that the plasma membrane was stained by comparison with the staining, imaging and colocalization of fluorescence protein-tagged membrane proteins in protoplasts from transgenic plant lines (data not shown).

\section{FM4-64}

The lipophilic FM4-64 dye incorporates into the outer leaflet of plasma membranes where it is emitting an intense fluorescence between $580 \mathrm{~nm}$ and $650 \mathrm{~nm}$. As soon as cell wall digestion was complete, the protoplasts were subsequently stained, using a final concentration of
$0.5 \%(\mathrm{v} / \mathrm{v})$ of the FM4-64 stock solution $(1 \mu \mathrm{g} / \mu \mathrm{l}$; see methods section). After an incubation period of 10$15 \mathrm{~min}$ at room temperature FM4-64 covered all areas of the plasma membrane homogenously (Figure 2A). Twenty to sixty minutes after the initial staining period, fluorescent particles became visible inside the cytoplasm. Occurring endocytosis events became more enhanced with ongoing incubation periods (Figure 2C). It has been reported that in tobacco BY2 cell suspension cultures as well as in BY2 protoplast suspensions (Nicotiana tabacum cultivar Bright Yellow 2 [32]) endocytosis events appeared 30 to 60 minutes after incubation with FM4-64. This is likely mediated by an active transport process since FM dyes cannot cross membranes due to their amphiphilic nature [33]. Ongoing 
endocytosis events at the plasma membrane indicated the protoplast's viability, which was confirmed by trypan blue treatment (Figure 3E). 30 minutes after incubation with FM4-64 an increasing number of fluorescent vesicles were detected inside the cytoplasm, indicating that endocytosis of the FM4-64 dye was still proceeding (Figure 2, C). FM4-64 is widely used as a non specific marker for endocytosis events and vesicle trafficking in living cells [34].

\section{LRB-PE}

The head group labeled phospholipid Lissamine Rhodamine B-Phosphoethanolamine (LRB-PE) has its excitation maximum at $557 \mathrm{~nm}$ whereas the emission maximum is at $583 \mathrm{~nm}$. In ternary lipid mixtures LRB$\mathrm{PE}$ was found to favor $\mathrm{L}_{\mathrm{d}}$-phases [35,36]. Cells were stained using $0.25 \%(\mathrm{v} / \mathrm{v})$ of the LRB-PE stock solution dissolved in DMSO $(1 \mu \mathrm{g} / \mu \mathrm{l}$; see methods section). Following an incubation period of 20 minutes at room temperature cells were subsequently imaged using onephoton microscopy. Upon LRB-PE treatment the plasma membrane exhibited a homogeneous stain (Figure 2E).

\section{DilC's and DiD}

In this study two DilCs, DiIC 12 and $\mathrm{DilC}_{18}$, and DiD were tested. In model membranes $\mathrm{DiIC}_{12}$ and $\mathrm{DiD}$ partitioned into $\mathrm{L}_{\mathrm{d}}$-phases, and $\mathrm{DiIC}_{18}$ partioned into both $\mathrm{L}_{\mathrm{d}^{-}}$and $\mathrm{L}_{\mathrm{o}}$-phases $[25,35,37]$. Both DiICs were dissolved in DMSO $(1 \mu \mathrm{g} / \mu \mathrm{l})$. Protoplasts were stained using final concentrations of $0.5 \%(\mathrm{v} / \mathrm{v})$ of $\mathrm{DiIC}_{12}$ and $\mathrm{DiIC}_{18}$ (see methods section). When used in protoplasts there were no preferred membrane regions for accumulation of either DiIC $_{12}$ (Figure 2G) or DiIC 18 (Figure 2I) molecules detectable. Both dyes augmented appeared in the cytoplasm within an hour, suggesting that $\mathrm{DiIC}_{12}$ as well as $\mathrm{DiIC}_{18}$ dyes underwent endocytosis events. In several staining experiments using different concentrations of $\mathrm{DiD}$ and expanding incubation times there were only weak fluorescence signals detectable. Again there was no plasma membrane compartmentalization observed using DiD (Figure 2K).

\section{BD-SM FL C 12 (BD-SM)}

To counter-stain $\mathrm{L}_{\mathrm{o}}$-phases a fluorescent sphingolipid analogue was employed, since i) lipids with mostly saturated hydrocarbon chains like sphingomyelins showed a preferred partitioning into $\mathrm{L}_{\mathrm{o}}$-phases in model membranes [25] and ii) in DRM fractions obtained from plants sphingolipids were enriched [14]. For this reason the Bodipy Sphingomyelin FL $\mathrm{C}_{12}$ (BD-SM) was used to label $\mathrm{L}_{\mathrm{o}}$-phases. According to information supplied by the manufacturer the BD-SM lipid analogue has the same stereochemical conformation as native, biologically active sphingolipids. Therefore BD-SM molecules incorporate into the plasma membrane and mix up with native sphingolipids. Phase partitioning experiments confirmed a participation of BD-SM in the DRM fraction (Figure 3C). BD-SM was added right after the isolation and purification of Arabidopsis plasma membrane components. Following Triton X-100 treatment Bodipy fluorescence was detectable in the DRM-fraction, whereas FM4-64 and LRB-PE at the same time could not be detected using fluorescence microscopy (not shown). To label protoplasts a stock solution of $1 \mu \mathrm{g} / \mu \mathrm{l}$ BD-SM in DMSO was generated, using a final concentration of $1 \%$ (v/v) BD-SM/DMSO. In freshly isolated protoplasts BD-SM was homogenously distributed in the plasma membrane. The dye was hardly taken up into the cytoplasm within time periods of about $1 \mathrm{~h}$; intracellular Bodipy fluorescence signals increased 15 to 18 hours (h) after labelling (Figure 3A).

\section{Combined dye staining of protoplasts}

The visualization of different lipid environments is clarified by the simultaneous staining of spectrally different fluorescent dyes with each color specifically attached to a liquid ordered or to a liquid disordered separating lipid. In the above experiments on Arabidopsis protoplasts, the best staining results were obtained using FM4-64, LRB-PE and BD-SM. DiD only weakly incorporated into plasma membranes, whereas $\mathrm{DiIC}_{12}$ and $\mathrm{DiIC}_{18}$ were strongly taken up into the cytoplasm, resulting in a decrease of fluorescence along time. To ensure solid fluorescence signals FM4-64 as well as LRB-PE were used individually to stain phospholipid enriched areas of the plasma membrane. BD-SM was employed at the same time to counter-stain sphingolipid enriched compartments.

In freshly isolated protoplasts there was initially a homogeneous stain of plasma membrane lipids detectable using combinations of FM4-64 and BD-SM (Figure 5A-D). In the merge image a strong yellow color appeared for all areas of the plasma membrane, indicating a homogenous distribution of phospholipid- and sphingolipid-phases (Figure 5C). Excitation of FM4-64 molecules with the $543 \mathrm{~nm}$ laser caused some cytosolic structures to show autofluorescence (Figure 5A-E). As compared to the white light transmission image these structures were chloroplasts showing chlorophyll fluorescence at given wavelengths (Figure 5D-H). Macroscopically the protoplasts appeared intact (Figure 5). $15 \mathrm{~h}$ after cell wall removal, the protoplasts underwent a rearrangement of lipids in the bilayer, resulting in a polarization of the plasma membrane. At the dorsal side a region appeared where a depletion of the FM4-64 signal was evident (arrows in Figure 5E-G). The plasma membrane appeared no longer as homogenously stained (Figure 5G). Polarization was even more enhanced $20 \mathrm{~h}$ 
after digestion of the cell wall (Additional file 1: Figure S1). In the protoplasts depicted FM4-64 fluorescence was strongly depleted at distinct sites of the membrane (Additional file 1: Figure S1, A-C; E-G; arrows). Time dependent lipid polarization was confirmed by a correlation analysis resulting from dye specific fluorescence signals (Figure $5 \mathrm{M}$ and $\mathrm{N}$ ). In freshly isolated protoplasts green BD-SM and red FM4-64 molecules appeared to be colocalized in the plasma membrane as indicated by high Pearson and Spearman correlation coefficients, ranging from 0.74 to 0.76 and 0.80 to 0.82 (Figure $5 \mathrm{M}$ ). With increasing time after enzymatically digesting the cell wall the correlation coefficients strongly decreased to 0.11 and 0.15 in polarized regions of interest (ROI's) (Figure $5 \mathrm{~N}$; for polarization analyses see also Additional file 2: Figure S2 and methods), which indicated an accumulation of lipids of a distinct phase. FM4-64 molecules were progressively detectable within the cytosol in time, indicating active endocytosis events at the plasma membrane to happen (Figure 2A-C). Since there are good hints that cellular endocytosis events depend on the presence of membrane sterols in plants [38], FM4-64 treatment might have induced changes in the lipid environment and thus the localization and activity of integral PM proteins. However, such changes were not observed in A. thaliana root, epidermis and cortex cells [31]. To reliably assure that FM4-64 uptake did not influence lipid polarization, experiments were repeated using LRBPE. LRB-PE has been shown to be a stable plasma membrane marker that was hardly taken up into the cytosol (Figure 2E). Again a lipid polarization was detected $15 \mathrm{~h}$ after cell wall removal as protoplasts were coevally stained using LRB-PE and BD-SM (Figure 5,I-K; arrows). A correlation analysis of LRB-PE and BD-SM fluorescence signals resulted in a dramatic change of the values of the correlation coefficients, decreasing from 0.89 in unpolarized protoplasts down to 0.39 in polarized ones. By the trend, these numbers strengthened the fact that plasma membrane resident lipids polarize in a time dependent manner. Nevertheless, we conclude that the time dependent differences in the plasma membrane lipid distribution can better be quantified by a FM4-64/ $\mathrm{BD}-\mathrm{SM}$ treatment rather than by a combined staining with LRB-PE and BD-SM.

\section{FRAP-experiments on polarized protoplasts}

The usage of fluorescent dyes on A. thaliana protoplasts revealed time dependent lipid redistribution events to happen, resulting in a polarization of the plasma membrane. To verify a separation of different lipid species into liquid ordered/liquid disordered phases fluorescence recovery after photobleaching (FRAP) experiments were performed on polarized protoplasts to reveal if differences in these observed lipid domains might agree to mobility studies with different lipid composition. From unilamellar model membranes consisting of ternary mixtures of sphingomyelin, phosphatidylcholine (DOPC) and cholesterol, it was reported that the content of cholesterol determined lipid mobility in sphingomyelin enriched, liquid ordered phases. It was shown that an increase in cholesterol led to decreased lateral diffusion coefficients, likely as a consequence of lateral separation into a liquid ordered phase, enriched in cholesterol and sphingomyelin and a liquid disordered phase, predominantly containing DOPC [39]. Because of the dense packaging of sterols and sphingolipids it is assumed for in vivo systems that sterol-rich compartments reflect the features of liquid ordered domains in artificial membranes $[26,40]$. Based on these findings it was supposed that changes in the cholesterol content could be a possible tool for viable cells in tuning their membrane lipid dynamics [41]. In FRAP-studies on polarized protoplasts FM4-64 enriched and depleted regions were bleached out in the plane of the membrane (see methods for details). Protoplasts were successfully stained using final concentrations of 0.5\% FM4-64 (v/v) and 1.0\% BD-SM $(\mathrm{v} / \mathrm{v}$; see methods). In either case disc shaped regions with spot sizes of $1.5 \mu \mathrm{m}$ in diameter were bleached (Figure 6). FRAP-studies demonstrated that areas with originally low or no FM4-64 but high BD-SM staining exhibit sedate recovery rates of BM-SM compared to the more accelerated ones of FM4-64 in FM4-64 enriched areas that also have approximately equal amounts of BD-SM. A diffusion coefficient [D] of $8.4 \times 10^{-3} \mu \mathrm{m}^{2} / \mathrm{s}$ and mobile fraction of $84 \%$ (or $16 \%$ immobility, $n=4$ ) were observed and fitted in the FM4-64 enriched regions (Figure 6C). In contrast the mobility and mobile fraction were lower of the BD-SM in the areas with essentially no FM4-64 with a diffusion coefficient [D] of $8.01 \times 10^{-4} \mu^{2} / \mathrm{s}$ and mobile fraction of only $28 \%$ (72\% immobility) (Figure 6D $\mathrm{n}=4$ ). These numbers indicate that lipids in FM4-64 enriched environments have a higher percentage that are able to move much faster than lipids residing in FM4-64 depleted environments, which on average demonstrate little or no motion. FRAP-experiments emphasized the coexistence of at least two lipid phases in the plasma membranes of viable protoplasts.

\section{Laurdan}

To verify the lipid polarization data, two-photon microscopy was employed using Laurdan as fluorescent probe. Laurdan is an environmentally sensitive dye that shows a $50 \mathrm{~nm}$ emission red-shift in its fluorescence spectrum in polar solvents and in the phospholipid liquiddisordered phase, as membranes undergo phase transitions from gel to fluid due to altered water contents in the lipid bilayer [42-44]. These differences in the plasma 

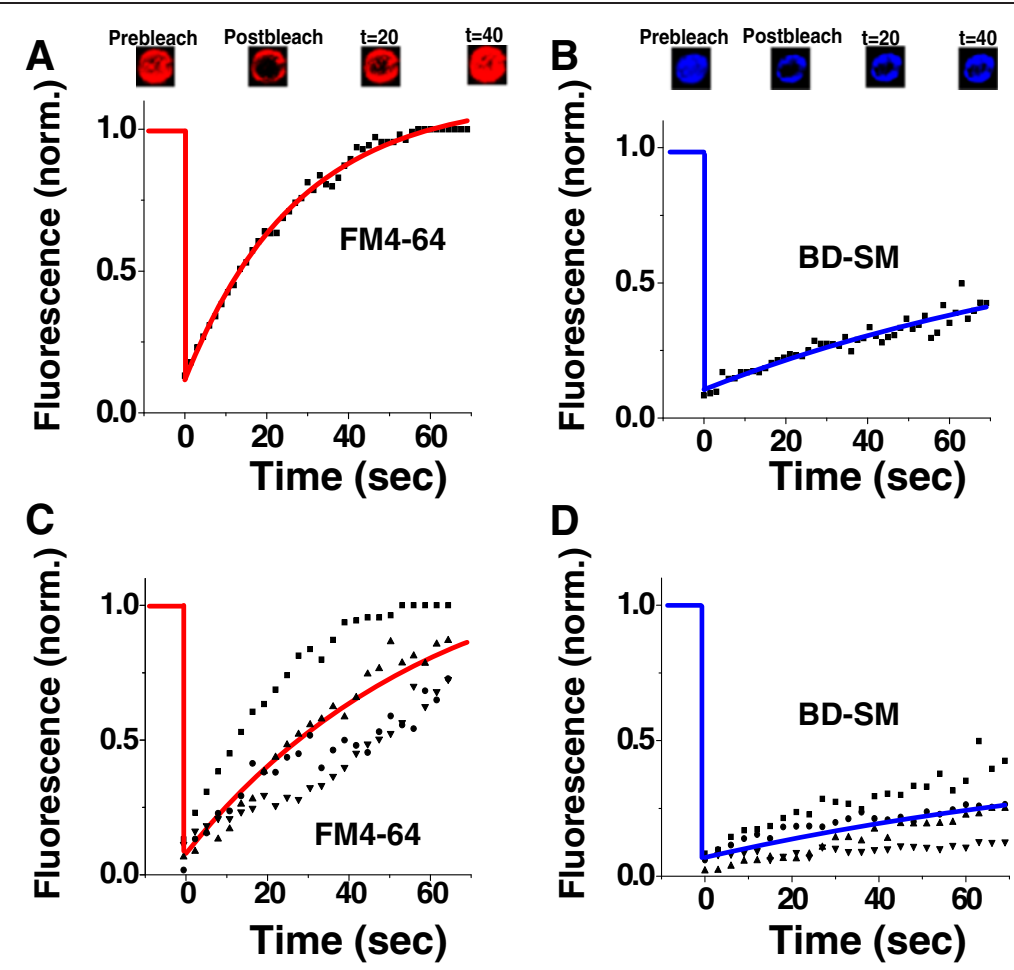

Figure 6 FRAP-experiments on polarized protoplasts. FRAP-experiments were performed on polarized areas of $15 \mathrm{~h}$ old protoplasts. (A) (Top) $10 \mu \mathrm{m} \times 10 \mu \mathrm{m}$ image of FM4-64 in an FM4-64 enriched region before, right after, $20 \mathrm{~s}$ and $40 \mathrm{~s}$ after the photobleach. (Bottom) Fluorescence recovery graph of FM4-64 from above protoplast region (data points as black squares) and with the recovery fitting (red line) depicting 100\% recovery or complete mobility and a diffusion coefficient of $0.124 \mu \mathrm{m}^{2} / \mathrm{s}$. (B) (Top) $10 \mu \mathrm{m} \times 10 \mu \mathrm{m}$ image of BD-SM in an FM4-64 depleted region before, right after, 20s and 40s after the photobleach. (Bottom) Fluorescence recovery graph of BD-SM from above protoplast region (data points as black squares) with a recovery fitting (blue line) depicting $48 \%$ recovery or a $48 \%$ mobile fraction and a diffusion coefficient of $3.4 \times 10^{-3} \mu \mathrm{m}^{2} / \mathrm{s}$ of the mobile fraction. (C \& D) Four typical FRAP-measurements are depicted for each dye with the single data point measurements represented as black circles, squares and triangles; Fit of the average of the four typical curves for the manuscript reported values of the mobile fractionand diffusion coefficients for (C) FM4-64 in FM4-64 enriched regions and (D) BD-SM in FM4-64 depleted regions. (C - red curve \& D - blue curve) (see manuscript). (See methods section for details of the FRAP acquisition and analysis.).

membrane water content can be visualized. Due to its molecular architecture Laurdans fluorescent naphthalene moiety exhibits a dipole moment between the 2-dimethylamino and the 6-carbonyl residues. Upon excitation the dipole moment increases; this increase is thought to cause a reorientation of surrounding solvent dipoles. The energy needed for the reorientation of surrounding solvents decreases the exited state energy of Laurdan and the emission spectrum of the probe is continuously red shifted when neighbored water molecules around reorganize [45]. Accordingly a red shift of the emission spectrum is observed in polar solvents, whereas in apolar solvents, such as in densely packed $\mathrm{L}_{\mathrm{o}}$-phases with decreased water content, the emission is more blueshifted. The false colored green images in Figure 4 reflect lipid ordered regions, whereas the red images reflect polar, lipid disordered sites of the plasma membrane with higher water content. To quantify specific shifts in the spectral behavior of Laurdan the generalized polarization (GP-) values were calculated $[44,46]$. The GP-value is dimensionless and reflects the predominant state of lipid order in lipid bilayers. In artificially generated ternary lipid mixtures liquid ordered domains have been characterized by high GP-values [46].

Protoplasts were stained immediately (Figure 4A-E), $15 \mathrm{~h}$ (Figure 4F-J) and $20 \mathrm{~h}$ (Figure 4K-O) after an enzymatic digestion of cell wall components. Directly after cell wall removal the plasma membrane fluorescence was homogenous, which indicated that polar and apolar lipid regions were equally distributed throughout the whole plasma membrane at this point (Figure 4A-D). A pixel to pixel calculation of the red and the green fluorescence signals revealed that there were no detectable differences in the lipid order, indicated by GP-values ranging from -0.3 to 0.2 (Figure 4E). This situation changed with increasing age of the protoplasts. $15 \mathrm{~h}$ after cell wall removal a rearrangement of plasma membrane lipids was detectable (Figure 4F-I). According to emitted Laurdan wavelengths the formation of two more blue shifted, apolar phases was observed (Figure 4G; arrows). The 
apolarity content within these phases was confirmed by according GP-values ranging from 0.2 up to 0.6 - indicating sites of increased lipid order (Figure 4J; arrows). $20 \mathrm{~h}$ after cell wall removal, polarization was even more enhanced (Figure 4K-O; arrows). At the lateral side of the cell a highly ordered lipid phase emerged (arrow in Figure 4O; GP-value between 0.6-0.8), whereas neighbored $\mathrm{PM}$ areas appeared to harbor less lipid ordered states (GP-values ranging from 0 to 0.2 ).

\section{Discussion}

Here we present simple staining protocols suited to label intact plant tissues and protoplasts using fluorescent probes of FM4-64, LRB-PE, DiIC 12 , DiIC $_{18}$, DiD, BD-SM and Laurdan. After initial incubations of Arabidopsis epidermal strips with either FM4-64 or BD-SM strong autofluorescence signals were detected that did not allow discriminations between dye-specific signals and unspecific ones coming from deeper leaf tissues (Figure 1). Some parts of the autofluorescence signal resulted from accumulations of the dyes within the cell wall microfibril texture. Therefore the plasma membrane participation of each dye in protoplasts was documented employing one- and two-photon microscopy. FM4-64 turned out to be a stable plasma membrane marker (Figure 2C), just as LRB-PE (Figure 2E) and BD-SM (Figure 3A). DiIC 12 (Figure 2G) and $\mathrm{DiIC}_{18}$ (Figure 2I) were strongly taken up into the cytosol which led to decreasing fluorescence signals at the plasma membrane over time. In contrast, a weak plasma membrane fluorescence was achieved using DiD (Figure 2K). The dye hardly incorporated into the bilayer. To detect possible differences in the lipid composition of Arabidopsis, protoplast dual staining experiments were performed (Figure 5). There, we found evidence for lipid polarization in the plasma membrane. Plasma membrane lipids underwent redistribution events within 15 to $20 \mathrm{~h}$ after cell wall digestion. Using BD-SM as marker for sphingolipid enriched membrane compartments and other fluorescent markers like FM464 and LRB-PE for phospholipid enriched areas, lipid redistributions could be visualized (Figure 5A-L). Lipid polarization was statistically allocated employing Pearson and Spearman correlation coefficients (Figure 5 $\mathrm{M}$ and $\mathrm{N}$; [47]). In freshly isolated protoplasts BD-SM and FM4-64 showed colocalization in the plasma membrane, displayed by high correlation coefficients (Figure 5M). Time dependent lipid polarization became evident by a strong decrease of the Pearson and Spearman correlation coefficients after $15 \mathrm{~h}$ (Figure $5 \mathrm{~N}$ ), which indicated a more random distribution of dye molecules within the plane of the membrane. To ensure that the dyes labeled inhomogeneous lipid phases the partitioning of BD-SM in the Arabidopsis DRM fraction was confirmed by Triton X-100 treatment of purified
Arabidopsis plasma membranes (Figure 3C). Detergent treatment revealed that BD-SM mixed up with natural phytosterols and sphingolipids, which are thought to self-aggregate to form lipid clusters. In DRMs isolated from Arabidopsis callus cultures sterols and sphingolipids showed a 4- to 5 -fold increase relative to the total plasma membrane $[14,48]$. In tobacco a similar increase of sterols and sphingolipids in DRMs was determined [49]. This indicated that BD-SM is likely labeling PM areas of elevated sterol/sphingolipid content in vivo. It was shown for sphingomyelins in model bilayer membranes that attached saturated acyl chains promoted the formation of raft-like lipid ordered phases that merged with each other to form larger domains [50].

Except for FM4-64 all dyes have been dissolved in dimethylsulfoxide (DMSO). DMSO/water or DMSO/buffer mixtures were used to stain Arabidopsis tissues and protoplasts. DMSO has been reported to have a strong influence on the structure of lipid membranes when used even at small mole concentrations, probably by displacing water and thereby modifying the structure of lipid bilayers [51]. Concerning cell biology, the amphiphilic DMSO molecule is known for its function to enhance membrane penetration, to induce cell fusion and for its role as cryoprotectant. Depending on DMSO concentration, membrane thickness can be affected, allowing for the formation of water pores to be induced and for the destruction of the bilayer structure of membranes [52]. In atomic-scale molecular dynamics simulations it was revealed for liquid disordered dipalmitoylphosphatidylcholine (DPPC/DMSO/water) systems that concentrations of $10-20 \mathrm{~mol} \%$ of DMSO led to pore formations within timescales of nanoseconds [52]. Here we used DMSO concentrations in the range of $0.25-3 \%(\mathrm{v} / \mathrm{v})$ which should not significantly alter the PM structure. Nevertheless there are reports using alfalfa protoplasts that a DMSO concentration of about $1 \%$ was already sufficient to induce changes in the transcript levels of certain genes, as reported for instance for cold acclimatization-specific (cas) genes. By increasing membrane rigidity, DMSO could possibly have induced calcium influxes, leading to a pronounced cold acclimation at room temperature [53]. Even so it cannot be ruled out that DMSO induced the expression of certain genes that could have influence on intracellular calcium concentrations as well as on the plasma membrane protein composition. In accompanied control experiments, however, there were no DMSOinduced effects on membrane polarization detectable when incubating Arabidopsis protoplasts for $24 \mathrm{~h}$ in buffer media containing up to $3 \%$ DMSO.

The FRAP-experiments left striking hints that there are at least two lipid populations resident in polarized poles of the plasma membrane. Lipid fractions that predominantly harbored fluorescent BD-SM molecules 
showed a significant decrease in recovery time compared to fractions in which FM4-64 was participating (Figure 6). The BD-SM labeled lipid fraction showed a diffusion coefficient [D] of $8.01 \times 10^{-4} \mu^{2} / \mathrm{s}$ with a mobile fraction of only $28 \%$ (a $72 \%$ immobile fraction), whereas for lipids in the FM4-64 labeled pool a [D] of $0.084 \mathrm{\mu m}^{2} / \mathrm{s}$ with an $84 \%$ mobile fraction (a $16 \%$ immobile fraction) was determined. These numbers indicated that lipids of the FM4-64 labeled fraction are able to move more than 10 times faster than lipids of the mobile BD-SM labeled fraction, and the FM4-64 was predominantly mobile, whereas the BDSM labeled fraction was predominantly immobile. In ternary systems consisting of cholesterol, sphingomyelin and dioleoylphosphatidylcholine (DOPC) diffusion coefficients were characterized with respct to lipid lateral diffusion rates. It was revealed that lipids of the $\mathrm{L}_{\mathrm{o}}$-phase appeared to recover 2-3 times slower than lipids of the $\mathrm{L}_{\mathrm{d}}$-phase [39]. In single gold particle tracking experiments on artificial giant unilamellar vesicles (GUV's) an increase in the sterol lipid content up to $50 \%$ of all lipids in planar lipid bilayers led to a nearly two fold reduction of [D] [54]. At given temperature [D] strongly depends on the movement of surrounding lipids as well as from the observation period. In the fluid, lipid disordered phase of ternary model membranes large diffusion coefficients of about $10^{-6} \mathrm{~cm}^{2} / \mathrm{s}$ have been reported at short time scales of $1 \mathrm{~ns}$, whereas at longer time scales diffusion coefficients decreased to $10^{-8}$ to $10^{-7}$ $\mathrm{cm}^{2} / \mathrm{s}[55,56]$. With an assumed [D] of $10^{-7} \mathrm{~cm}^{2} / \mathrm{s}$, an individual lipid would cover a lateral distance of about $6 \mathrm{~nm}$ within a time period of $1 \mu \mathrm{s}$. The applied FRAP-technique for the measurement of lateral diffusion rates of different lipid phases in viable protoplasts however did not allow time and length resolutions of this magnitude since this method is based on optical systems, whose spatial resolving power is limited by the chosen scan method and by the diffraction of light. Acquired FRAP datasets are, therefore, not directly comparable to diffusion coefficients calculated from atomistic simulations mimicking lipid diffusions in model membranes [55]. Nevertheless, there have been efforts made in defining lipid diffusion coefficients in model membranes employing FRAP; in pure liquid disordered, dimyristoylphosphatidylcholine (DMPC) systems a [D] of $7.5 \times 10^{-8} \mathrm{~cm}^{2} / \mathrm{s}$ was measured at $35^{\circ} \mathrm{C}$, decreasing to $6.0 \times$ $10^{-8} \mathrm{~cm}^{2} / \mathrm{s}$ when temperature dropped to $26^{\circ} \mathrm{C}$. As cholesterol was added to the same system the liquid ordered phase formed; at $35^{\circ} \mathrm{C}$ [D] was measured to be $3.0 \times 10^{-8}$ $\mathrm{cm}^{2} / \mathrm{s}$ in the $\mathrm{L}_{\mathrm{o}}$-phase, being further reduced to $1.8 \times 10^{-8}$ $\mathrm{cm}^{2} / \mathrm{s}$ at $26^{\circ} \mathrm{C}$ [57]. FRAP measurements in protoplasts were carried out at room temperatures of about $20-22^{\circ} \mathrm{C}$. Taking the pure numbers, lipid diffusion coefficients in protoplasts appear to be two to three orders of magnitude slower compared to those found in artificial membranes.

To our best knowledge no similar FRAP-datasets are available describing lateral lipid diffusion coefficients in viable Arabidopsis protoplasts to this date. In analogy to findings in model membranes it is assumable for polarized protoplasts that the slower fluorescence recovery rates of BD-SM phases are caused by an accumulation of sterols at distinct sites of the plasma membrane (Figure 5G-K; Figure 6).

Different dye loadings and FRAP-experiments revealed plasma membrane lipid heterogeneity in Arabidopsis protoplasts. One further possibility to prove this finding was the employment of a lipid environment sensitive fluorescent probe like Laurdan. Since in $\mathrm{L}_{\mathrm{o}}$-phases sterols and sphingolipids are tightly packed these regions have a less water content compared to $L_{d}$-phases, in which lipids are more densely packed. Laurdan can be applied to visualize such differences in the water content. In Experiments on A. thaliana protoplasts, a final Laurdan concentration of $5 \mathrm{mM}$ was enough to successfully stain viable plasma membranes. For mammalian cells in contrast concentrations in the micromolar range are used. This might be explained by the more complex sterol profile of plant plasma membranes.

In $\mathrm{L}_{\mathrm{o}}$-phases fluorescence is shifted into the more blue spectrum of light (false colored green in Figure 4), in polar $\mathrm{L}_{\mathrm{d}}$-phases accordingly more into the red. Immediately after cell wall removal there were no lipid phase polarizations detectable (Figure 4A-C), confirmed by a calculation of the general polarization (GP-) value in a pixel to pixel analysis of Laurdan images (Figure 4E). The GP-value can be used as indicator for the water content of lipid phases and accordingly as a degree for the predominant state of lipid order [43]. A GP-value of -1 indicates an aqueous phase, whereas a GP-value of +1 indicates a fully ordered phase. GP-values in Figure $4 \mathrm{E}$ ranged from -0.3 to 0.2 . Experimentally these values depend on the lipid composition and on temperature. In the liquid disordered phase of model membranes GP-values ranged from -0.3 to 0.3 while in liquid ordered phases these values are typically in the range of 0.5 to 0.6 [45]. In liposomes with equal molecular ratios of DOPC, cholesterol and sphingomyelin the GP-values of the liquid disordered phase ranged from -0.05 to 0.25 whereas a GP of 0.25 to 0.55 indicated the coexistence of a liquid ordered phase [42]. It has been reported that "GP-values of living cells may not always directly correspond to those obtained in artificial membrane systems", but that GP-values allow comparisons in order to rule out fluidity differences within plasma membranes [42]. Recently Laurdan has been used to examine the degree of lipid order in different tissues of vertebrates. In vital zebrafish embryos the 
quantification of membrane order ranged between GPvalues of 0 to 0.2 in more ordered apical membranes compared to basolateral membranes exhibiting a decreased lipid order with GP-values of 0 to 0.13 [58]. In mammalian MDCKII and RAW264.7 cell lines GP-values ranged from -0.1 to 0.3 . Using the steroldepleting agent methyl- $\beta$-cyclodextrin GP-values decreased with decreasing sterol content of the plasma membrane, examined by a fluorescent live imaging approach [59]. Using Laurdan on native BY2 plasma membranes GPvalues dropped from $0.65\left(\right.$ at $4^{\circ} \mathrm{C}$ ) to 0.55 (at $22^{\circ} \mathrm{C}$ ) down to 0.30 (at $40^{\circ} \mathrm{C}$ ). In analogy to findings in model membranes the BY2 plasma membrane constitution would accordingly be consisting of gel-like, liquid ordered domains below $20^{\circ} \mathrm{C}(\mathrm{GP}>0.55)$; above this temperature the relative order of the membrane progressively decreased [60].

Laurdan treatments of Arabidopsis protoplasts revealed that $15 \mathrm{~h}$ after cell wall removal redistributions of lipid phases occur (Figure 4F-H). With respect to the Laurdan fluorescence characteristics there were lipid phases with different water content emerging (Figure 4F and G). GP-values of up to 0.6 indicated high levels of lipid order for regions of the plasma membrane (Figure 4J, arrows). After $20 \mathrm{~h}$ polarization was even more pronounced, resulting in one distinct lipid ordered pole covering the lateral side of the protoplast (Figure $4 \mathrm{~K}-\mathrm{O}$, arrows). Likely this pole arose from the coalescence of smaller regions of lipid order, as seen in Figure 4J. For model membranes it has been reported that $\mathrm{L}_{\mathrm{o}}$-phases, predominantly harboring lipids with saturated acyl chains like sterols and sphingomyelins, merged with each other to form larger domains [50].

Lipid polarization could be confirmed by several independent non-invasive tests like staining experiments with different lipid analogues (Figure 5A-L), FRAPmeasurements (Figure 6) and Laurdan labeling of plasma membranes including a calculation of GP-values (Figure 4). At this point it is unclear what exactly caused plasma membrane resident lipids to relocate into distinct poles. Polarizations of lipid domains are reported for several biological systems across species, especially during cytokinesis [61]. Experiments in fission yeast (Schizosaccharomyces pombe) revealed that there are sterols enriched at the growing cell tips and at sites of cytokinesis [62]. In pollen tubes lipid microdomain polarization was found to be essential for polarized tube growth involving reactive oxygen species (ROS) signaling. ROS-producing NADPH oxidases were reported to be associated with DRMs and to depend on the presence of sterols [63]. ROS signaling plays important roles in plants since reactive oxygen species help controlling processes of growth, development, biotic and abiotic stress response and programmed cell death [64]. In the experiments performed here there were in contrast neither cell divisions nor any kinds of developmental processes detectable during observation periods of up to $24 \mathrm{~h}$.

It has also previously been reported that plant protoplasts start regenerating their cell walls in suited cultivation media. In Nicotiana tabacum protoplast cell wall regeneration started within 30 minutes after removing cell wall components, and cell wall regeneration processes were indicated by formations of cellulose microfibril depositions at distinct sites at the protoplasts surface [65]. The spatial organization of cellulose microfibrils defines the cell wall texture and is achieved by an oriented microfibril deposition. Transmembrane cellulose-synthase-complexes are directly linked to components of the cytoskeleton and enable an organized deposition of cellulose microfibrils [66]. Some of the cell wall building components like chitin- and $ß$-D-glycan-synthases have been shown to be resident in detergent resistant membrane domains of Oomycetes [67]. Callose and cellulose synthases have also found to be strongly enriched in DRM fractions isolated from plasma membranes of the tree species Populus, indicating that active proteins involved in cell wall biosynthesis associate with sphingolipid/sterol enriched, lipid ordered phases in vivo [68]. It is likely that lipid redistributions in plasma membranes of Arabidopsis protoplasts reflect cellular attempts to regenerate absent cell walls. In tobacco protoplast cell walls fully regenerated within a time period of up to six hours. Observations were made, that the period in which the regeneration processes finished, strongly depended on the method that was used before when isolating the protoplasts; depending on the isolation procedure the regeneration processes could take up to 16 hours [65]. Nevertheless there were no indicators for cell wall regenerations found in Arabidopsis protoplasts during observation periods of up to $24 \mathrm{~h}$. This could be due to the medium used for cultivating the protoplasts; the medium still contained intact proteolytic enzymes like cellulases and pectolyases, which could have hindered cell wall regenerations. In Convolvus arvensis protoplasts it was shown that the ability of single cells to regenerate their cell walls was strongly diminished in the presence of active proteolytic enzymes [69].

\section{Conclusions}

The basic knowledge in terms of lipid behavior derived from experiments in artificial membranes of defined lipid mixture. In viable cell membranes this knowledge is limited, since there are still missing links concerning lipid behavior and lipid composition. Lipophilic fluorescent dyes and lipid analogues could help broadening our knowledge from model membranes to functional ones. So far a majority of the emerging bulk of commercial available dyes and lipids suited for fluorescent real time imaging is designed to stain mammalian cells and is because of the altered lipid composition - not suited for 
the use in plants. Designing dyes and lipids basically for the usage in mammalian cells surely is owed by the fact that plant lipidomics have so far been of limited interest only. Within the last years there are trends indicating that this situation is changing. Here we took attempts to adapt some of the mammalian staining protocols to viable plant cells and documented the plasma membrane localization of FM4-64, LRB-PE, DiIC $12, \mathrm{DiIC}_{18}, \mathrm{DiD}$, BD-SM and Laurdan in Arabidopsis protoplasts. Optimizing the staining protocols we found evidence that there are different lipid phases emerging in the plasma membranes of protoplasts $15-20 \mathrm{~h}$ after an enzymatic digestion of the cell wall. Dual labeling, as well as, FRAP experiments in polarized plasma membrane areas and Laurdan based GP-value calculations confirmed this assumption. The most plausible explanation seems that these lipid polarizations are reflecting cellular efforts to restore the cell walls of protoplasts. Nevertheless more experiments are needed to discover the molecular origin of this redistribution of lipids. Plant-adapted staining protocols included here might alleviate future approaches to investigate lipid compositions in viable plant cells. The use of fluorescent dyes and lipid analogues suited for confocal laser scanning microscopy and related techniques enables the study of lipid dynamics and lipid distributions in viable cells in real time.

\section{Methods}

One and two photon microscopy

Images were obtained using confocal microscopes (Zeiss LSM5 Pascal; Carl Zeiss Microimaging, Jena, Germany or TCS SP5; Leica, Mannheim, Germany). For two-photon microscopy approaches a femtosecond pulsed Ti:Sa-laser (Mai Tai, Spectra Physics; Darmstadt, Germany) was utilized, coupled into a TCS SP5 system (Leica) (Table 1).

\section{Whole tissue staining}

Epidermis strips of adult A. thaliana leaves were stripped off of the whole leaf and kept in water. The utilization of epidermal strips helped reducing background autofluorescence signals and further enabled a full tissue penetration by employed laser beams. For proper staining experiments dyes were either solved

\section{Table 1 LSM-Filterset/TCS SP5 Emission settings}

\begin{tabular}{llll}
\hline & Exc. (nm) & Emm. $(\mathbf{n m})$ & Emm. max. $(\mathbf{n m})$ \\
\hline FM4-64 & 543 & $580-650$ & 640 \\
LRB-PE & 543 & $580-630$ & 583 \\
DilCs & 543 & $560-620$ & 565 \\
DiD & 543 & $640-680$ & 665 \\
BD-SM & 488 & $500-550$ & 520 \\
\hline
\end{tabular}

water (FM4-64; $1 \mu \mathrm{g} / \mu \mathrm{l})$ or in dimethylsulfoxide (BD$\mathrm{SM} ; 1 \mu \mathrm{g} / \mu \mathrm{l})$. Based on the stocks individual aqueous staining solutions were prepared. Arabidopsis epidermal strips were incubated for 15 minutes with FM4-64 at a final concentration of $1.0 \%(\mathrm{v} / \mathrm{v})$ or with BD-SM (up to 30 minutes at a final concentration of $5.0 \% \mathrm{v} / \mathrm{v}$ ). Directly after staining the strips were shortly incubated in pure water to wash away excessive dye molecules.

\section{Single dye staining of protoplasts FM4-64}

\{N-(3-triethylammoniumpropyl)-4-(6-(4-(diethylamino) phenyl)hexatrienyl)pyridium dibroide?

The lipophilic FM4-64 dye is based on a polyethylene structure. The dye is water soluble and non toxic for living cell tissues according to manufacturers' information (Invitrogen; Karlsruhe, Germany); the viability of the cells was additionally confirmed by trypan blue treatment (Figure 3E). The dye was solved in pure water to a stock solution of $1 \mu \mathrm{g} / \mu \mathrm{l}$. In staining experiments a final concentration of $0.5 \%(\mathrm{v} / \mathrm{v})$ was used to stain Arabidopsis protoplasts. After an incubation period of 10 to 15 minutes at room temperature in buffer medium cells were fairly stained. A wavelength of $543 \mathrm{~nm}$ was employed to excite the fluorophore; the emission spectrum of FM4-64 was in the range of 580-650 nm (emission max.: $640 \mathrm{~nm}$ ).

\section{Lissamine rhodamine B-phosphoethanolamine (LRB-PE)}

\{1,2-dimyristoyl-sn-glycero-3-phosphoethanolamine-N(lissaminerhodamine B sulfonyl)\}

LRB-PE (Avanti Polar Lipids; Alabaster, USA) was solved in dimethylsulfoxide (DMSO) to a stock concentration of $1 \mu \mathrm{g} / \mu \mathrm{l}$. Protoplasts were incubated for 20 minutes at room temperature at a final concentration of $0.25 \%(\mathrm{v} / \mathrm{v})$ LRB-PE/DMSO in protoplast buffer. An excitation wavelength of $543 \mathrm{~nm}$ was used to excite the fluorophore; emitted light was collected between 580 and $630 \mathrm{~nm}$ (emission max.: 583).

DilC $_{12} \&$ DilC $_{18}$

$\left\{\right.$ DiIC $_{12}$ : $\quad\left(1,1^{\prime}\right.$-didodecyl-3,3,3', $3^{\prime}$-tetramethyl-indocarbocyanine perchlorate)\}

$\left\{\right.$ DiIC $_{18}:\left(1,1^{\prime}\right.$-dioctadecyl-3,3,3', $3^{\prime}$-tetramethyl-indocarbocyanine perchlorate)\}

Both lipophilic DiIC dyes (Invitrogen) were solved in DMSO to a stock concentration of $1 \mu \mathrm{g} / \mu \mathrm{l}$. Protoplasts were incubated using a final concentration of $0.5 \%(\mathrm{v} / \mathrm{v})$ $\mathrm{DiIC}_{12} / \mathrm{DMSO}$ or rather $\mathrm{DiIC}_{18} / \mathrm{DMSO}$ in protoplast buffer. After an incubation time of 20 minutes at room temperature protoplasts were scanned with the laser scanning microscopes, employing a wavelength of $543 \mathrm{~nm}$ to excite the fluorophores; emission was collected between 560 and $620 \mathrm{~nm}$ (emission max: 565). 


\section{DiD}

$\left\{\left(1,1^{\prime}\right.\right.$-dioctadecyl-3,3,3', $3^{\prime}$-tetramethylindodicarbocyanine 4-chlorobenzenesulfonate salt)\}

For the application of the lipophilic DiD dye (Invitrogen) a stock concentration of $1 \mu \mathrm{g} / \mu \mathrm{l}$ in DMSO was generated. To label protoplast suspensions various concentrations were generated, ranging between 0.25 and 3.0\% DiD/DMSO (v/v). Using different DiD concentrations only weak fluorescence signals were detectable at the plasma membrane (along with increased incubation periods of up to 30 minutes). DiD was excited using a $543 \mathrm{~nm}$ laser, showing its maximal emission at $665 \mathrm{~nm}$.

\section{Bodipy-sphingomyelin FL $\mathrm{C}_{12}$ (BD-SM)}

$\{\mathrm{N}$-(4,4-difluoro-5,7-dimethyl-4-bora-3a,4a-diaza-s-indacene-3-dodecanoyl)sphingosyl phosphocholine)\}

The amphiphilic BD-SM lipid analogue consists of a sphingosine moiety which is linked to a fatty acid by an amide bond. The sphingomyelin itself is covalently linked to a Bodipy fluorophor (Invitrogen). Referring to information of the manufacturer BD-SM features the same stereochemical conformation than biologically active sphingomyelins, in spite of the fluorescence labeling. The bodipy fluorophore exhibits similar emission and excitation wavelengths like the green fluorescent protein, GFP (exc.: $488 \mathrm{~nm}$, em. 500-550 nm). The dye was solved in DMSO to a stock concentration of $1.0 \mu \mathrm{g} / \mu \mathrm{l}$. Protoplasts were stained using $1 \%(\mathrm{v} / \mathrm{v})$ of the stock solution in protoplast buffer. After an incubation time of 20 minutes at room temperature cells were fairly stained.

\section{Combined dye staining of protoplasts}

To label putative different lipid phases, protoplast suspensions were co-incubated using stocks of FM4-64, LRB-PE and BD-SM (all solved in DMSO to $1 \mu \mathrm{g} / \mu \mathrm{l}$ ). In dual labelling experiments the protoplasts were coevally incubated using 0.5\% FM4-64 (v/v) and 1\% BD-SM (v/v) or LRB-PE $(0.25 \% \mathrm{v} / \mathrm{v})$ and BD-SM $(1 \% \mathrm{v} / \mathrm{v})$. Protoplasts were incubated for 20 minutes at room temperature and subsequently imaged.

\section{Correlation analysis}

Correlation analyses were performed using the colocalization plug-in for Image J (v. 137.c), as developed by French et al. [47]. The plug-in allowed a performance of quantitative statistical colocalization on two-color confocal images. For each protoplast two regions of interest (ROI) were chosen and the Pearson and Spearman correlation coefficients calculated (an example of a correlation analysis is given in Additional file 2: Figure S2). For polarized protoplasts one ROI (ROI1) was unpolarized or was enriched with both lipid-dyes and the other region (ROI2) was the predominately depleted FM4-64 region. If the items are correlated, they will have a value $>0.1$ to 1 . If the colors are uncorrelated (meaning separated), they will have a value of $<-0.1$ to -1 . If the colors are randomly correlated or if only one color appears within a region, the value is essentially zero or approximately $>-0.1$ and $<0.1$. The Spearman coefficient was calculated in addition to the one of Pearson, since it does not assume the linear relationship of statistic variables. The usage of both coefficients to determine possible correlations allowed for higher statistic significance. All correlation coefficients are displayed as box-charts, each one showing the highest/lowest individual correlation value, the standard error bar for the values displayed, the average correlation value and the standard deviation for each ROI.

\section{Laurdan}

\{6-lauroyl-2-dimethylaminonaphthalene)\}

Laurdan was dissolved to $60 \mathrm{mM}$ in a solution of DMSO containing $17 \%$ ethanol (|>97\%). The final concentration in a distinct protoplast suspension was $5 \mathrm{mM}$; after incubation times of up to 60 minutes at room temperature cells were sufficiently stained. To generate desired excitation wavelengths of about $390 \mathrm{~nm}$ a multiphoton (MP-) laser at $760 \mathrm{~nm}$ (Mai Tai, Spectra Physics) was employed. Blue light was gathered between 400 to $440 \mathrm{~nm}$ (Intensity blue); the red shifted spectrum was collected using a bandpass ranging from 490 to $550 \mathrm{~nm}$ (Intensity red).

\section{Pixel by pixel analysis}

To quantify changes in the Laurdan emission spectra pixel to pixel analyses of the blue and the red signals were performed. The predominant lipid order in different areas of the plasma membrane was measured as "generalized polarisation" (or GP-) value, following the equation

$$
\left(\mathrm{I}_{\mathrm{b}}\right)-\left(\mathrm{I}_{\mathrm{r}}\right) /\left(\mathrm{I}_{\mathrm{b}}\right)+\left(\mathrm{I}_{\mathrm{r}}\right)=\mathrm{GP} .
$$

$I_{b}$ and $I_{r}$ correspond to the intensities of the blue and the red emission spectrum [46]. Liquid ordered regions show high GP-values since these regions are enriched in sterols. An enrichment in sterols lead to poor water levels. The membrane gets apolar and Laurdan emission and excitation wavelengths are more shifted into the blue spectrum. The GP-value is dimensionless, ranging from -1 (less ordered phase, polar lipid surrounding) to +1 (fully ordered phase, apolar lipid surrounding).

\section{Arabidopsis thaliana protoplast isolation}

Leaves of 8 to 10 week old $A$. thaliana col 0 plants were cut into small sections $(1 \times 1 \mathrm{~cm})$ and were incubated for 
$2 \mathrm{~h}$ in digestion buffer at $28^{\circ} \mathrm{C}$ in an incubation shaker (30 rpm). The digestion buffer contained cellulase $(0.8 \%$ w/v; Onozuka R10; Onozuka, Yakult Pharmaceutical Industry; Tokyo, Japan), pectolyase $(0.1 \% \mathrm{w} / \mathrm{v}$; Sigma Aldrich; Taufkirchen, Germany), bovine serum albumin $(0.5 \% \mathrm{w} / \mathrm{v})$ and polyvinylpyrolidone $(0.5 \% \mathrm{w} / \mathrm{v})$; finally calcium chloride was added $(1 \mathrm{mM})$. The osmolarity was adjusted to $280 \mathrm{milliosmol} / \mathrm{kg}$ using sorbitol; the $\mathrm{pH}$ was adjusted to 5.6 (MES-Tris). After incubation a short centrifugation step was necessary $\left(80-100 \mathrm{rpm}, 10 \mathrm{~min}, 4^{\circ} \mathrm{C}\right)$ to separate undigested leaf fragments from the protoplasts.

\section{Cell viability}

Trypan blue stain (0.4\%; Lonza; Walkersville, USA) was used as an indicator for cell viability. Trypan blue was solved to $1 \mathrm{mg} / \mathrm{ml}$ in $0.6 \mathrm{M}$ mannitol. Nonviable cells absorb the dye and appear blue while intact cells and membranes remain unstained. The dye was directly applied into the protoplast buffer, and the suspension was carefully mixed. To obtain a good black-white contrast suited for imaging Trypan blue concentrations of up to $20 \%(\mathrm{v} / \mathrm{v})$ were used. After 10 minutes incubation at room temperature cells were sufficiently stained.

\section{DRM-isolation}

Detergent-resistant membranes from plasma membranes were generated from Arabidopsis thaliana leaves as described elsewhere $[49,70,71]$. Before the extraction of the DRM-fraction by using $1 \% \mathrm{v} / \mathrm{v}$ Triton X-100, BD-SM $(1.0 \mu \mathrm{g} / \mu \mathrm{l})$ was added to the purified plasma membrane. BD-SM and plasma membrane lipids were mixed until the suspension cleared up. Subsequently $1 \%$ Triton X-100 was applied (optimized as determined in [49]), followed by a $16 \mathrm{~h}$ sucrose density centrifugation. The DRM-fraction was extracted and aliquots were used for confocal microscopy studies.

\section{FRAP acquisition and analysis}

The FRAP-data were acquired with either the Leica LASAF FRAP Wizard or Zeiss LSM5 Pascal FRAP program, under the imaging conditions listed above. The bleach area on the surface of the plasma membrane was always a $1.5 \mu \mathrm{m}$ diameter disc. The data could be acquired every 0.25 seconds but were reduced to $1.5 \mathrm{sec}-$ onds between analysis points for the purposes of this manuscript. The images were analyzed with Image J (NIH, USA) and the data was exported to Origin (Origin Labs, USA). Average fluorescence intensities within ROIs in the bleached regions were analyzed for each detection channel to obtain the recovery data. Corrections were made for photobleaching during scanning by monitoring neighboring cells and analyzing their signals. Mobile fractions were calculated and the recovery was fit to the equation: $\mathrm{I}=\mathrm{A} 1-\mathrm{A} 2 * \exp \left(-\mathrm{k}^{*} \mathrm{t}\right)$ where $\mathrm{I}$ is the average intensity, $\mathrm{k}$ is the rate of the exponential recovery, $\mathrm{t}$ is the time, A1 is the full recovery value and A2 is the value of the drop in intensity after the bleach. The lateral diffusion coefficients, $\mathrm{D}$, were determined from $D=\left(\frac{\beta \omega^{2}}{4 \ln 2}\right) k$, where $\beta$ is the bleach depth (determined to be 1.2), $\omega$ is the radius of the bleach area and $k$ is the fitted rate constant.

\section{Additional files}

Additional file 1: Figure S1. FM4-64/BD-SM staining of protoplasts. FM4-64/BD-SM staining on protoplasts, $20 \mathrm{~h}$ post cell wall removal. Polarization was even more enhanced after this time period $(A-H)$. (A; E) FM4-64 fluorescence. (B; F) BD-SM fluorescence. In the merged images clear-cut polarizations were detected ( $C ; G$, arrows). Tranmission images (D; H).

Additional file 2: Figure S2. Example for correlation analyses. For statistically relevant correlation analyses two ROl's within the plasma membranes of protoplasts were chosen and the Pearson and Spearman correlation coefficients calculated from confocal images (see methods section). The images selected are of (A) an unpolarized protoplast from Figure 5, C and two $15 \mathrm{~h}$ old protoplasts (B) one with FM4-64 staining from Figure 5, G and (C) one with LRB staining from Figure 5, K. (A - C) ROI1 was placed into non-polarized membrane areas and served as negative control for putative correlation. (A) ROI2 reflects the correlation of green BD-SM and red FM4-64 fluorescence signals next to ROI1 in unpolarized regions in unpolarized protoplasts. (B \& C) In 15 h old protoplasts ROI2 was exclusively placed to ROI1 neighboring polarized areas. High correlation coefficients indicated a colocalization of pixels of the two different colors (green and red), whereas negative correlation coefficients indicated a separation of the two colors.

\section{Competing interests}

The authors declare that they have no competing interests.

\section{Authors' contributions}

JOB developed new and modified existing protocols suited for staining viable plant tissues and protoplasts; performed SP- \& MP-microscopy; analyzed acquired digital images; interpreted the data and drafted the manuscript. FD isolated Arabidopsis DRM-fractions and provided support during staining experiments. IF helped coordinating the experiments and gave useful advice. GSH conceived of the study and its design; drafted parts of the manuscript and was an advisory capacity in terms of confocal imaging. $\mathrm{RH}$ assisted in conceiving the study and provided advice regarding the results. GSH, FD \& IK helped correcting the manuscript, which final version all authors have read and approved.

\section{Acknowledgements}

We thank Prof. Martin J. Müller for his advice on artificial lipid analogues and Prof. Werner Kaiser for being in an advisory capacity for major and minor points of plant biochemistry. We thank Claudia Horntrich for her assistance with protoplast preparations and maintenance of transgenic plant lines. This work was funded by the German Science Foundation (DFG) grants GK 1342 for the Graduate College of Lipid Signaling in Plants and for FZ-82 for the Rudolf Virchow Center of Biomedicine to GSH and JOB, by a Howard Hughes Medical Institute Grant to GSH and the University of Würzburg funding program for Open Access Publishing.

\section{Author details}

${ }^{1}$ Institute for Molecular Plant Physiology and Biophysics, University Würzburg, Julius-von-Sachs Platz 2, D-97082 Würzburg, Germany. ${ }^{2}$ Microscopy Group, Rudolf Virchow Center, University of Würzburg, Josef Schneider Str. 2, D15, D-97080 Würzburg, Germany. ${ }^{3}$ Present address: Institute of Neuro- and Sensory Physiology, Düsseldorf University Hospital, Universitätsstr. 1, D-40225 
Düsseldorf, Germany. ${ }^{4}$ Departments of Biology and Physics, Wilkes University, 84 W. South St., Wilkes-Barre, PA 18766, USA.

Received: 9 March 2012 Accepted: 20 July 2012

Published: 6 August 2012

\section{References}

1. Foster $L$, Hoog CLD, Mann M: Unbiased quantitative proteomics of lipid rafts reveals high specificity for signaling factors. Proc Natl Acad Sci USA 2003, 100:5813-5818.

2. Raffaele S, Bayer E, Lafarge D, Cluzet S, Retana SG, Boubekeur T, LeborgneCastel N, Carde JP, Lherminier J, Noirot E, Satiat-Jeunemaitre B, LarocheTraineau J, Moreau P, Ott T, Maule AJ Reymond P, Simon-Plas F, Farmer EE, Bessoule JJ, Mongrand S: Remorin, a solanaceae protein resident in membrane rafts and plasmodesmata, impairs potato virus $\mathrm{X}$ movement. Plant Cell 2009, www.plantcell. org/cgi/doi/10.1105/tpc.108.064279.

3. Simons K, Ikonen E: Functional rafts in cell membranes. Nature 1997, 387:569-572.

4. Brown DA, London E: Structure and function of sphingolipid- and cholesterol-rich membrane rafts. J Biol Chem 2000, 275:17221-17224.

5. Brown DA, Rose JK: Sorting of GPI-anchored proteins to glycolipidenriched membrane subdomains during transport to the apical cell surface. Cell 1992, 68:533-544.

6. Lingwood D, Simons K: Detergent resistance as a tool in membrane research. Nat Protoc 2007, 2:2159-2165.

7. Lichtenberg D, Goni FM, Heerklotz H: Detergent-resistant membranes should not be identified with membrane rafts. Trends Biochem Sci 2005, 30(8):430-436.

8. Zappel NF, Panstruga R: Heterogeneity and lateral compartmentalization of plant plasma membranes. Curr Opin Plant Biol 2008, 11:632-640.

9. Tanner W, Malinsky J, Opekarova M: In plant and animal cells, detergentresistant membranes do not define functional membrane rafts. Plant Cell 2011, www.plantcell.org/cgi/doi/10.1105/tpc.111.086249.

10. Simons K, Gerl MJ: Revitalizing membrane rafts: new tools and insights. Nat Rev Mol Cell Biol 2010, 11(10):688-699.

11. Malinsky J, Opekarova M, Tanner W: The lateral compartmentation of the yeast plasma membrane. Yeast 2010, 27(8):473-478

12. Grossmann G, Opekarova M, Malinsky J, Weig-Meckl I, Tanner W: Membrane potential governs lateral segregation of plasma membrane proteins and lipids in yeast. EMBO J 2007, 26(1):1-8.

13. Lillemeier BF, Pfeiffer JR, Surviladze Z, Wilson BS, Davis MM: Plasma membrane-associated proteins are clustered into islands attached to the cytoskeleton. PNAS 2006, 103:18992-18997.

14. Borner GHH, Sherrier DJ, Weimar T, Michaelson LV, Hawkins ND, MacAskill A Napier JA, Beale MH, Lilley KS, Dupree P: Analysis of detergent-resistant membranes in arabidopsis. evidence for plasma membrane lipid rafts. Plant Physiol 2005, 137:104-116.

15. Tsui-Pierchala BA, Encinas M, Milbrandt J, Johnson EM Jr: Lipid rafts in neuronal signaling and function. Trends Neurosci 2002, 25(8):412-417.

16. Kierszniowska S, Seiwert B, Schulze WX: Definition ofarabidopsis sterolrich membrane microdomains by differential treatment with methylb-cyclodextrin and quantitative proteomics. Mol Cell Proteomics 2009, 8(4):612-623.

17. Bhat RA, Panstruga R: Lipid rafts in plants. Planta 2005, 223:5-19.

18. Beck JG, Mathieu D, Loudet C, Buchoux S, Dufourc EJ: Plant sterols in "rafts": a better way to regulate membrane thermal shocks. FASEB J 2007, Express Article fj.06-7809com.

19. Uemura M, Joseph RA, Steponkus PL: Cold acclimation of arabidopsis thaliana.Plant Physiol 1995, 109:15-30.

20. Sperling P, Franke S, Lüthje S, Heinz E: Are glucocerebrosides the predominant sphingolipids in plant plasma membranes? Plant Physiol Biochem 2005, 43:1031-1038.

21. Ahmed SN, Brown DA, London E: On the origin of sphingolipid/ cholesterol-rich detergent-insoluble cell membranes: physiological concentrations of cholesterol and sphingolipid induce formation of a detergent-insoluble, liquid-ordered lipid phase in model membranes. Biochemistry 1997, 36:10944-10953.

22. Schroeder RE, London E, Brown DA: Interactions between saturated acyl chains confer detergent resistance on lipids and glycosylphosphatidylinositol (GPI)-anchored proteins: GPI-anchored proteins in liposomes and cells show similar behavior. PNAS 1994, 91:12130-12134.
23. Silvius JR: Role of cholesterol in lipid raft formation: lessons from lipid model systems. Biochim Biophys Acta 2003, 1610:174-183.

24. Vist MR, Davis JH: Phase equilibria of cholesterol/ dipalmitoylphosphatidylcholine mixtures: ${ }^{2} \mathrm{H}$ nuclear magnetic resonance and differential scanning calorimetry. Biochemistry 1990, 29:451-464.

25. Simons K, Vaz WL: Model systems, lipid rafts, and cell membranes. Annu Rev Biophys Biomol Struct 2004, 33:269-295.

26. McMullen TPW, Lewis RNAH, McElhaney RN: Cholesterol-phospholipid interactions, the liquid-ordered phase and lipid rafts in model and biological membranes. Current Opinion in Colloid and Interface Science 2004, 8:459-468.

27. Ipsen JH, Mouritsen OG, Zuckermann MJ: Theory of thermal anomalies in the specific heat of lipid bilayers containing cholesterol. Biophys J 1989, 56:661-667.

28. Ipsen JH, Karlstrom G, Mouritsen OG, Wennerstrom H, Zuckermann MJ: Phase equilibria in the phosphatidylcholine-cholesterol system. Biochim Biophys Acta 1987, 905:162-172.

29. Lingwood D, Kaiser HJ, Levental I, Simons K: Lipid Rafts as functional heterogeneity in cell membranes. Biochem Soc Trans 2009, 37:955-960

30. Hancock JF: Lipid Rafts: contentious only from simplistic standpoints. Nat Rev Mol Cell Biol 2006, 7:456-462.

31. Jelinkova A, Malinska K, Simon S, Kleine-Vehn J, Parezova M, Pejchar P, Kubes M, Martinec J, Friml J, Zazimalova E, Petrasek J: Probing plant membranes with FM dyes: tracking, dragging or blocking? Plant J 2010, 61:883-892.

32. Nagata T, Nemoto Y, Hasezawa S: Tobacco BY-2 cell line as the "HeLa" cell in the cell biology of higher plants. Int Rev Cytol 1992, 132:1-30.

33. Bolte $S$, Talbot C, Boutte $Y$, Catrice O, Read ND, Satiat-Jeunemaitre B: FMdyes as experimental probes for dissecting vesicle trafficking in living plant cells. Journal Of Microscopy 2004, 214(2):159-173.

34. Fischer-Parton S, Parton RM, Hickey PC, Dijksterhuis J, Atkinson HA, Read ND: Confocal microscopy of FM4-64 as a tool for analysing endocytosis and vesicle trafficking in living fungal hyphae. J Microsc 2000, 198:246-259.

35. Juhasz J, Davis JH, Sharom FJ: Fluorescent probe partitioning in giant unilamellar vesicles of 'lipid raft' mixtures. Biochem J 2010, 430:415-423.

36. Baumgart T, Hunt G, Farkas ER, Webb WW, Feigenson GW: Fluorescence probe partitioning between $L_{o} / L_{d}$ phases in lipid membranes. Biochim Biophys Acta 2007, 1768:2182-2194

37. Kahya N: Light on fluorescent lipids in rafts: a lesson from model membranes. Biochem J 2010, 430:e7-e9.

38. Samaj J, Baluska F, Voigt B, Schlicht M, Volkmann D, Menzel D: Endocytosis, actin cytoskeleton, and signaling. Plant Physiol 2004, 135:1150-1161.

39. Filippov A, Orädd G, Lindblom G: Lipid lateral diffusion in ordered and disordered phases in raft mixtures. Biophys J 2004, 86:891-896.

40. Baumgart T, Hammond AT, Sengupta P, Hess ST, Holowka DA, Baird BA, Webb WW: Large-scale fluid/fluid phase separation of proteins and lipids in giant plasma membrane vesicles. PNAS 2007, 104(9):3165-3170.

41. Kahya N, Scherfeld D, Bacia K, Poolman B, Schwille P: Probing Lipid mobility of Raft-exhibiting model membranes by fluorescence correlation spectroscopy. J Biol Chem 2003, 278(30):28109-28115.

42. Gaus K, Gratton E, Kable EP, Jones AS, Gelissen I, Kritharides L, Jessup W: Visualizing lipid structure and raft domains in living cells with twophoton microscopy. PNAS 2003, 100:15554-15559.

43. Parasassi T, Krasnowska EK, Bagatolli L, Gratton E: Laurdan and prodan as polarity-sensitive fluorescent membrane probes. I Fluoresc 1998, 8(4):365-373.

44. Parasassi T, Gratton E, Yu W, Wilson P, Levi M: Two photon fluorescence microscopy of LAURDAN generalized polarization domains in model and natural membranes. Biophys J 1997, 72:2413-2429.

45. Sanchez SA, Tricerri MA, Gunther G, Gratton E: Laurdan generalized polarization: from cuvette to microscope. Modern Research and Educational Topics in Microscopy (Formatex) 2007, 2:1007-1014.

46. Dietrich C, Bagatolli LA, Volovyk ZN, Thompson NL, Levi M, Jacobson K, Gratton E: Lipid rafts reconstituted in model membranes. Biophys J 2001, 80:1417-1428.

47. French AP, Mills S, Swarup R, Bennett MJ, Pridmore TP: Colocalization of fluorescent markers in confocal microscope images of plant cells. Nat Protoc 2008, 3(4):619-628. 
48. Lynch DV, Dunn TM: An introduction to plant sphingolipids and a review of recent advances in understanding their metabolism and function. New Phytol 2004, 161:677-702.

49. Mongrand S, Morel J, Laroche J, Claverol S, Carde JP, Hartmann MA, Bonneu M, Simon-Plas F, Lessire R, Bessoule JJ: Lipid rafts in higher plant cells. The Journal Of Biological Chemistry 2004, 279(35):36277-36286.

50. Samsonov AV, Mihalyov I, Cohen FS: Characterization of cholesterolsphingomyelin domains and their dynamics in bilayer membranes. Biophys J 2001, 81:1486-1500.

51. Gordeliy VI, Kiselev MA, Lesieur P, Pole AV, Texeira J: Lipid membrane structure and interactions in dimethyl sulfoxide/water mixtures. Biophys $J$ 1998, 75:2343-2351.

52. Gurtovenko AA, Anwar J: Modulating the structure and properties of cell membranes: the molecular mechanism of action of dimethyl sulfoxide. J Phys Chem B 2007, 111:10453-10460.

53. Örvar BL, Sangwan V, Omann F, Dhindsa RS: Early steps in cold sensing by plant cells: the role of actin cytoskeleton and membrane fluidity. Plant J 2000, 23(6):785-794.

54. Ladha S, Mackie AR, Harvey LJ, Clark DC, Lea EJA, Brullemans M, Duclohier $H$, Lateral Diffusion in Planar Lipid Bilayers: A fluorescence recovery after photobleaching investigation of its modulation by lipid composition cholesterol, or alamethicin content and divalent cations. Biophys J 1996, 71:1364-1373.

55. Apajalahti T, Niemelä P, Govindan PN, Miettinen MS, Salonen E, Marrink SJ, Vattulainen I: Concerted diffusion of lipids in raft-like membranes. Faraday Discuss 2010, 144:411-430.

56. Niemela P, Ollila S, Hyvonen MT, Karttunen M, Vattulainen I: Assessing the nature of lipid raft membranes. PLOS Comput Biol 2007, 3:304-312.

57. Almeida PFF, Vaz WLC, Thompson TE: Lipid diffusions, free area, and molecular dynamics simulations. Biophys J 2005, 88:4434-4438.

58. Owen DM, Magenau A, Majumdar A, Gaus K: Imaging membrane lipid order in whole, living vetrebrate organisms. Biophys J 2010, 99:L07-L09.

59. Wheeler G, Tyler KM: Widefield microscopy for live imaging of lipid domains and membrane dynamics. Biochim Biophys Acta 2011, 1808:634-641.

60. Roche Y, Gerbeau-Pissot P, Buhot B, Thomas D, Bonneau L, Gresti J, Mongrand S, Perrier-Cornet JM, Simon-Plas F: Depletion of phytosterols from the plasma membrane provides evidence for disruption of lipid rafts. FASEB J 2008, 22(11):3980-3991.

61. Echard $A$ : Membrane traffic and polarization of lipid domains during cytokinesis. Biochem. Soc. Transact. 2008, 36(3):395-399.

62. Wachtler V, Rajagopalan S, Balasubramanian MK: Sterol-rich plasma membrane domains in the fission yeast Schizosaccharomyces pombe. J Cell Sci 2003, 116:867-874

63. Liu P, Li R, Zhang L, Wang QL, Niehaus K, Baluska F, Samaj J, Lin JX: Lipid microdomain polarization is enquired for NADPH oxidase-dependent ROS signaling in Picea meyeri pollen tube tip growth. Plant J 2009. doi:10.1111/j.1365-313X.2009.03955.x.

64. Bailey-Serres J, Mittler R: The roles of reactive oxygen species in plants. Plant Physiol 2006, 141:311.

65. VanAmstel TNM, Kengen HMP: Callose deposition in the primary wall of suspension cells and regenerating protoplasts, and its relationship to patterned cellulose synthesis. Can JBot 1996, 74:1040-1049.

66. Paredez AR, Somerville CR, Ehrhardt DW: Visualization of cellulose synthase demonstrates functional association with microtubules. Science 2006, 312(5779):1491-1495.

67. Briolay A, Bouzenzana J, Guichardant M, Deshayes C, Sindt N, Bessueille L, Bulone V: Cell wall polysaccharide synthases are located in detergentresistant membrane microdomains in oomycetes. Appl Environ Microbiol 2009, 75(7):1938-1949.

68. Bessueille L, Sindt N, Guichardant M, Djerbi S, Teeri TT, Bulone V: Plasma membrane microdomains from hybrid aspen cells are involved in cell wall polysaccharide biosynthesis. Biochem J 2009, 420:93-103.
69. Horine RK, Ruesink AW: Cell wall regeneration around protoplasts isolated from convolvulus tissue culture. Plant Physiol 1972, 50:438-445.

70. Larsson K: Lipid phase transitions in membranes involving intrinsic periodic curvature. Chem Phys Lipids 1988, 49(1-2):65-67.

71. Yoshida S, Uemura M, Niki T, Sakai A, Gusta LV: Partition of membrane particles in aqueous two-polymer phase system and its practical use for purification of plasma membranes from plants. Plant Physiol 1983, 72(1):105-114.

doi:10.1186/1746-4811-8-28

Cite this article as: Blachutzik et al:: Methods of staining and visualization of sphingolipid enriched and non-enriched plasma membrane regions of Arabidopsis thaliana with fluorescent dyes and lipid analogues. Plant Methods 2012 8:28.

\section{Submit your next manuscript to BioMed Central and take full advantage of:}

- Convenient online submission

- Thorough peer review

- No space constraints or color figure charges

- Immediate publication on acceptance

- Inclusion in PubMed, CAS, Scopus and Google Scholar

- Research which is freely available for redistribution

Submit your manuscript at www.biomedcentral.com/submit
C) Biomed Central 